\title{
Panorama sobre enfoques y tendencias en la enseñanza del español en las dos últimas décadas *
}

\section{Overview of approaches and trends in Spanish language teaching over the past two decades}

\author{
Nour Adoumieh Coconas \\ Instituto Superior de Formación Docente Salomé \\ Ureña, República Dominicana \\ nour.adoumieh@isfodosu.edu.do
}

\author{
Luisa María Acosta Caba \\ Instituto Superior de Formación Docente \\ Salomé Ureña, República Dominicana \\ luisa.acosta@isfodosu.edu.do
}

Palabras claves: enseñanza, lengua, español, enseñanza de idiomas, revisión sistemática de la literatura.

\section{Resumen}

Los enfoques y metodologías en la enseñanza de las disciplinas son dinámicos y dependen de una multiplicidad de factores, entre los cuales podríamos destacar el contexto del grupo, así como el dominio de competencias pedagógicas, específicas, tecnológicas e incluso informacionales del docente. El objetivo de esta revisión sistemática de la literatura es analizar las tendencias y enfoques metodológicos en la enseñanza de la lengua española como lengua materna y segunda lengua (L1/L2). El corpus estuvo constituido por 1661 artículos científicos exportados de las bases de datos Scopus, Web of Science y SciELO en el periodo 2001- 2020. La información obtenida en estas bases de datos fue sistematizada y analizada con ayuda de Zotero 5.0 y MAXQDA 2020. En primera instancia se suprimieron los duplicados y luego de un proceso de selección, se analizaron los artículos finales bajo algunos criterios como: año, país, tema y problema abordado, contexto de aplicación, enfoques y metodologías de la lengua. Los resultados arrojados evidenciaron que la mayor producción se encuentra en España y EEUU; se enfatiza en la Educación Superior; los temas se relacionan con el desarrollo de las competencias y los enfoques más empleados son el comunicativo y el sociocultural. Se privilegia el aprendizaje por tareas y basado en proyectos, así como las metodologías enfocadas en el uso de la tecnología.

Recepción: Sep 26 de 2020 | Aceptación: Oct 28 de 2020 | Publicación: Dic 15 de 2020

\footnotetext{
* Esta publicación constituye un avance del proyecto de investigación financiado por el Instituto Superior de Formación Docente Salomé Ureña. PI-012-2019 Concepciones de la didáctica de la lengua desde la perspectiva de los estudiantes de la Licenciatura de Primaria Segundo Ciclo del ISFODOSU.
} 


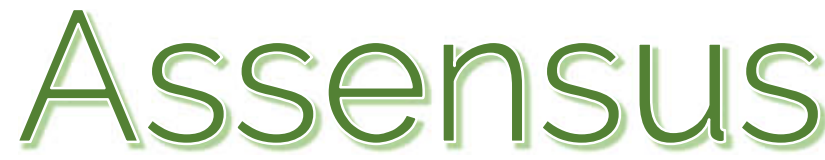

Revista de Investigación educativa y pedagógica

\begin{tabular}{ll}
\hline & Abstract \\
& The approaches and methodologies in the teaching of the \\
disciplines are dynamic and depend on a multiplicity of factors, \\
among which we could highlight the context of the group and the \\
domain of pedagogical, specific, technological and even \\
informational skills of the teacher. The objective of this \\
systematic review of the literature is to explain the trends and \\
methodological approaches in the teaching of the Spanish \\
language as a mother tongue and second language (L1/L2). The \\
corpus consisted of 1661 scientific articles exported from the \\
Scopus, Web of Science and SciELO databases in the period \\
2001-2020. The information obtained in these databases was \\
systematized and analyzed with the help of Zotero 5.0 and \\
MAXQDA 2020. In In the first instance, duplicates were \\
eliminated and after a selection process, the final articles were \\
analyzed under some criteria such as: year, country, topic and \\
problem addressed, context of application, approaches and \\
methodologies of the language. The results obtained showed that \\
the highest production is found in Spain and the US; it is \\
emphasized in Higher Education; The topics are related to the \\
development of competencies and the most used approaches are \\
communicative and sociocultural. Task-based and project-based \\
Keywords: \\
teaching, \\
language, \\
Spanish, language \\
teaching, \\
systematic review are privileged, as well as methodologies focused on the \\
use of technology.
\end{tabular}

of the literature.

Received: Sep 26, 2020 | Accepted: Oct 28, 2020 | Published: Dec 15, 2020 


\section{Introducción}

La didáctica de la lengua se ha convertido en un foco de interés en el marco de los procesos de revisión y actualización curricular que se han venido dando en los últimos años, tanto en República Dominicana como a nivel internacional (Cisneros Estupiñán et al., 2016; García y Matos Moquete, 2013; Haché y Montenegro, 2013). Este interés parte de la intención del sistema educativo de los distintos países de ajustar los procesos de enseñanzaaprendizaje a la educación basada en competencias, realidad que se evidencia en los diseños curriculares y planes de estudio de los niveles inicial, primario, secundario y superior (García Retana, 2011; Ministerio de Educación de la República Dominicana, 2016; Tobón, 2006, 2007). Específicamente en el área de lengua, estas intenciones se traducen en los esfuerzos por aplicar el enfoque comunicativo, funcional y textual. Según las propuestas de infinidad de autores, este enfoque conduce a la conformación de un usuario de la lengua capaz de participar efectivamente en las prácticas comunicativas de los diversos ámbitos en los que interactúa (Arnáez Muga, 2006; Cassany et al., 2003; Lomas García, 1999; Prado Aragonés, 2004).

Existe una gran preocupación por los bajos resultados que obtiene un alto porcentaje de la población estudiantil en los desempeños en el área de lengua. Así lo han mostrado los resultados de las pruebas PISA (Grupo Iberoamericano de PISA, 2009; Instituto de Evaluación del Ministerio de Educación y Ciencia, 2007; Ministerio de Educación, Cultura y Deporte, 2014, 2016; Ministerio de Educación de España, 2010; OECD, 2019) y los estudios regionales comparativos PERCE, SERCE y TERCE (Casassus et al., 1998; Flotts et al., 2015; Valdés et al., 2008). Esto parece indicar que la práctica educativa podría estar todavía muy alejada de las implicaciones pedagógicas de estos enfoques y tendencias actuales en la enseñanza del español para lograr ciudadanos competentes comunicativamente. No obstante, el estudio ERCE 2019, realizado recientemente por la Organización de las Naciones Unidas para la Educación, la Ciencia y la Cultura y la Oficina Regional de Educación para América Latina y el Caribe (2020), revela en sus resultados que al menos los lineamientos curriculares de los países participantes, incluyendo la República Dominicana, están acordes con el abordaje de la enseñanza de la lengua desde los principios teóricos y metodológicos de la didáctica específica del área. Lo expuesto anteriormente apunta a la necesidad de disminuir la brecha entre el ser y el deber ser en la enseñanza de la lengua española.

El interés por realizar una revisión sistemática de la literatura acerca de las tendencias y enfoques metodológicos en la enseñanza del español surge en el marco del proyecto de investigación "Concepciones de la didáctica de la lengua desde la perspectiva de los estudiantes de la Licenciatura en Primaria Segundo Ciclo del ISFODOSU", Instituto Superior de Formación Docente Salomé Ureña. En la actualidad, con miras a elevar los indicadores de calidad en investigación, las universidades exigen que sus profesores publiquen. Desde 
aproximadamente los últimos cinco años, las publicaciones se han incrementado exponencialmente y el campo de la Lingüística Aplicada a la enseñanza del español no ha sido la excepción (Bolívar, 2020; Harzing y Alakangas, 2016). En consecuencia, se considera importante hacer una revisión sistemática de los artículos relacionados con el tema para detectar aportes que contribuyan con una comprensión más organizada y amplia del estado del arte.

Por otra parte, lograr una metodología de enseñanza de la lengua alineada con el enfoque comunicativo, funcional, textual y discursivo supone necesariamente analizar las tendencias y enfoques metodológicos empleados para enseñar el español, ya sea como lengua materna o como segunda lengua. Para el estudio del estado actual de las investigaciones existentes, se identificaron artículos de revistas indexadas publicados desde el 2001 hasta el 2020 en las bases de datos Scopus, Web of Science y SciELO. En tal sentido, realizar una revisión sistemática de la literatura señalada se convierte en la mejor vía para:

a) dotar el corpus recuperado de una estructura coherente que permita comprender cuáles son las tendencias y enfoques metodológicos empleados en L1/L2

b) detectar vacíos que ameritan ser llenados en cuanto al conocimiento científico sobre las tendencias y enfoques metodológicos para enseñar lengua española

c) valorar la viabilidad de la aplicación de estos enfoques y tendencias

d) identificar hallazgos útiles para mejorar la praxis pedagógica y así definir la ruta de investigaciones futuras.

Esta revisión sistemática deja las puertas abiertas a futuras revisiones que incluyan otras bases de datos que aporten mayor visibilidad a estudios realizados en el contexto latinoamericano donde se aborde la enseñanza del español únicamente como lengua materna.

\section{Referentes teóricos}

\section{Enfoques de enseñanza de la lengua}

Para tener un panorama general de las investigaciones en el área de la didáctica de la lengua, es necesario contar con una base teórica acerca de los enfoques y tendencias metodológicas que se aplican en distintos contextos de enseñanza del español. En lo que respecta al enfoque de enseñanza, se refiere al modo de abordaje de la práctica educativa y orienta el quehacer pedagógico para alcanzar unos determinados resultados de aprendizaje. En otras palabras, cómo se aprende y cómo se enseña (Ministerio de Educación de la República Dominicana, 2019). En la didáctica de la lengua, existen enfoques muy variados, entre los que cabe señalar el enfoque comunicativo, cuyo origen se remonta a los años 70 en 
el contexto de la enseñanza de lenguas extranjeras. Su finalidad es el desarrollo de la competencia comunicativa de los usuarios la cual comprende el componente lingüístico, el sociolingüístico y el pragmático (Consejo de Europa, 2003). Dentro de sus principios generales se encuentran el uso de textos auténticos, el favorecimiento de situaciones de comunicación, el texto como eje de los procesos de comprensión y producción, el abordaje integrado de las destrezas escuchar, hablar, leer y escribir, la gramática al servicio de la comunicación y la lectura y la escritura como prácticas sociales (Ministerio de Educación de la República Dominicana, 2019).

Otro enfoque destacado en la enseñanza de la lengua es el sociocultural, nacido de las teorías propuestas por Lev Vygotsky, el cual destaca el valor del entorno social en que interactúa el sujeto que aprende. Dicho enfoque tiene implicaciones en el ámbito de la enseñanza de la lengua en lo que respecta al aprendizaje de los discursos que median las prácticas sociales y a la apropiación de la cultura donde se inserta el sujeto que aprende.

Por su parte, el enfoque democrático alude a prácticas de enseñanza de la lengua en las que se consideran "conceptos esencialmente controvertidos como: democracia, ciudadanía, género, clase social y pluralismo cultural” (Grossman, 2008, p. 46). Además, se puede identificar el enfoque ecléctico, el cual se concibe desde los procesos de alfabetización, cuyos resultados en adultos y niños dependerá de la funcionalidad de los contenidos y su propensión a satisfacer las necesidades inmediatas del estudiante (Simich-Dudgeon, 2009).

En lo concerniente al enfoque integrado, Llovet Vila (2006) afirma que este surgió como respuesta a la necesidad de adecuar la enseñanza formal de las lenguas extranjeras a las competencias reales de los usuarios en el contexto europeo, empleando la lengua objeto de aprendizaje para impartir cualquier materia. Según Rodríguez Arocho (2018), el enfoque de alfabetización crítica se origina gracias a los aportes de Vygotsky, Paulo Freire e Ignacio Martín Baró, a partir del cual se considera la alfabetización como una actividad situada en la historia, mediada por la cultura y ejecutada en un contexto social.

\section{Metodologías de enseñanza de la lengua}

Los enfoques de enseñanza de la lengua se concretizan por medio de metodologías, entre las que se pueden considerar aquellas que están basadas en los estilos de aprendizaje, tales como el aprendizaje basado en tareas, el aprendizaje de la lengua en comunidad y el aprendizaje integrado de contenido y lenguaje. Enseñar lengua según esta metodología implica ajustar el quehacer docente a las características de los alumnos para el logro de los aprendizajes esperados (Gutiérrez Tapias, 2018). En adición, se encuentran las tendencias metodológicas que involucran la tecnología, las cuales se presentan bajo múltiples formas que impactan positivamente la enseñanza y el aprendizaje de la lengua, gracias a la incorporación de herramientas que posibilitan la comunicación en varias lenguas (Trujillo Sáez et al., 2019). 
Existen también tendencias metodológicas centradas en la autorregulación, entendida como un conjunto de estrategias que favorecen la autonomía, la motivación y la autoeficacia en el aprendizaje de las lenguas, al tiempo que contribuyen con la mejora en el desempeño de los usuarios al momento de escuchar, hablar, leer y escribir (Noreña Peña, 2019). Otras tendencias metodológicas relacionadas con la autorregulación son aquellas que enfatizan en el discurso y en el desarrollo de las competencias retóricas. Las primeras buscan el desarrollo de la competencia discursiva, es decir, que el usuario sea capaz de desenvolverse adecuadamente en determinadas situaciones de comunicación produciendo e interpretando textos orales y/o escritos (Centro Virtual Cervantes, 2009a). Las segundas aluden a las habilidades que tienen los sujetos para operar estratégicamente con las pistas textuales al momento de comprender (Sánchez et al., 2002).

Otra tendencia metodológica en la enseñanza de la lengua es la secuencia didáctica, definida por Camps (2003) como un conjunto de actividades interrelacionadas y organizadas en torno a una situación que les da sentido y que favorece el desarrollo de las habilidades comunicativas. Por otra parte, la transposición didáctica aboga por la transferencia de los saberes propios de las disciplinas científicas de referencia al contexto de la enseñanza de la lengua, cuya finalidad es que dichos saberes, más allá de ser enseñados, sean empleados en situaciones diversas (Álvarez Angulo, 1998).

\section{Metodología}

En consonancia con lo definido por Fink (2010), una revisión de la literatura se resume como una metodología sistemática, explícita y duplicable que sintetiza el abordaje y la proyección de un tema a través de un conjunto de investigaciones publicadas en revistas científicas. En este sentido, para garantizar la rigurosidad de pasos y generar un producto confiable, seguimos las recomendaciones establecidas en la Declaración PRISMA (Urrútia y Bonfill, 2010) y en Rädiker y Kuckartz (2020). Estos últimos autores presentan su propuesta fundamentada en las investigaciones de Fink (2010), Hart (2017), Gough, Oliver y Thomas (2017) y Heyvaert, Hannes y Onghena (2016).

En una primera fase, se determinó el objetivo, analizar las tendencias y enfoques metodológicos en la enseñanza de la lengua española bien sea como lengua materna o como segunda lengua (L1/L2). Para ello, se trazaron las siguientes preguntas de investigación: ¿cuál es la productividad diacrónica evidenciada en el corpus de estudio sobre la enseñanza de la lengua?, ¿cuáles son los principales países y contextos de aplicación de los estudios analizados?, ¿cuáles son los principales objetos de estudio y problemas tratados? y ¿cuáles son las tendencias y enfoques metodológicos empleados en la enseñanza de la lengua española (L1/L2)?. Luego, se seleccionaron tres bases de datos: Scopus, Web of Science y 
SciELO. Se consideraron los criterios de calidad, visibilidad y oportunidad de descarga en formato RIS a fin de poder posteriormente facilitar el proceso de codificación. Se excluyó de las bases de datos a Google Scholar, dado que, a pesar de presentar información vinculada con citación, rescata de manera indiscriminada todo tipo de publicación. De esta forma, se evitaría la desigualdad en cuanto al género discursivo artículo académico (Fricke, 2018). Además, realizar un tamizaje manual representaba una desventaja en tiempo. Otra base de datos excluida fue Redalyc, ya que dificultaba el rescate de los metadatos de los artículos, aunque posee valiosas publicaciones que exteriorizan la producción intelectual del tema en América Latina.

Una vez seleccionadas las bases de datos y después de varias pruebas de operadores, se limitó la búsqueda a "Language AND teaching AND Spanish" OR “Approaches AND Teaching AND Language" en el periodo 2001 - 2020, idiomas en inglés y español y áreas de Lengua y Educación. El total de artículos recabados fue de 1661, de los cuales 1081 fueron de Scopus, 500 de Web of Science y 80 de SciELO. Los 1661 artículos fueron importados al gestor de referencias Zotero 5.0, a través del cual se eliminaron 197 duplicados. Seguidamente, se constató la correspondencia de los documentos importados con el objetivo de investigación y se eliminaron 880. El total de documentos que conformaron el corpus después de este filtro fue de 584. Estos fueron importados a MAXQDA 2020, donde se les asignó un peso mediante el acuerdo de intercodificadores, quedando excluidos 180 por abordar el español como lengua heredada y fenómenos lingüísticos descontextualizados del ámbito de la enseñanza. A continuación, se presenta el gráfico 1 que ilustra los detalles del proceso:

Gráfico 1. Procedimiento para conformar el corpus de análisis

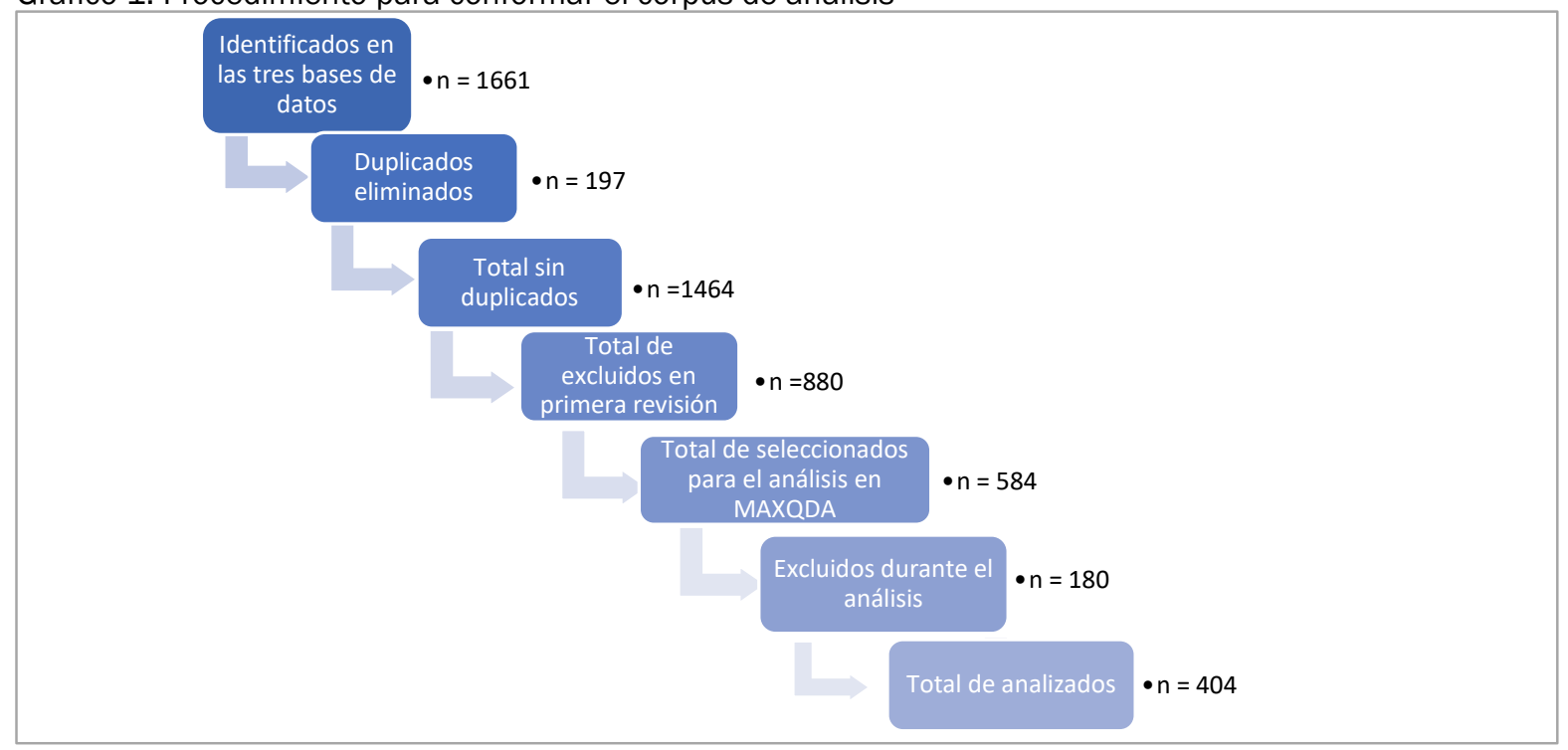

Fuente: Elaboración propia a partir de los datos procesados en Zotero 5.0 y MAXQDA 2020 
El corpus resultante, constituido por los 404 artículos, fue analizado en MAXQDA 2020 atendiendo a una exploración primaria, a partir frecuencias, nubes y palabras clave que permitió generar una serie de códigos basados en la categorización inductiva y posteriormente fueron ampliados a través de la codificación axial propia de la teoría fundamentada (Strauss et al., 2002). Paralelo a ello, se ejecutó el proceso de codificación, identificación de coocurrencias, frecuencias y tendencias temáticas, problemáticas y metodologías didácticas. Es importante destacar que los criterios de inclusión y exclusión de los artículos fueron: abordar la enseñanza del español como primera o segunda lengua, contemplar aportes didácticos, evidenciar explícitamente un enfoque de enseñanza y/o alguna metodología didáctica de manera aplicada o reflexiva y excluir investigaciones de español como lengua heredada.

Por último, se analizó el contenido de los artículos según las categorías codificadas y los segmentos discursivos seleccionados. Se registró un total de 229 categorías conceptuales alrededor de 6 dimensiones analíticas. Esta revisión sistemática se centró en el análisis cuantitativo de: i) productividad diacrónica, ii) país de la investigación, iii) contexto de aplicación, iv) temática abordada, v) problemática frecuente y vi) enfoques y metodologías recurrentes. Para el análisis cualitativo, se consideraron las tres últimas dimensiones. Con el fin de garantizar la calidad de la investigación y evitar los puntos ciegos o por solapamiento que pueden generarse, se siguió la directriz de Flick (2014) y se socializó el proyecto con pares académicos.

\section{Resultados}

Los estudios seleccionados en esta revisión sistemática realizada en tres bases de datos de relevancia científica como lo son Scopus, Web of Science y SciELO exhibieron un alto índice de productividad diacrónica en el área de la enseñanza del español. Luego de un proceso riguroso de selección, a través de los 404 artículos elegidos para el análisis cuantitativo y cualitativo en el rango 2001 al 2020, se observó una tendencia ascendente en las publicaciones. Este planteamiento concuerda con Price (1986), quien estipula como ley que la información científica suele duplicarse cada 10 a 15 años, lo cual se puede visualizar en el gráfico 2. Esta situación permite reflexionar sobre el interés en el tema y que además los países de aplicación han sido diversos. Los 404 estudios estuvieron circunscritos a 37 países. Entre los 10 primeros están: España 34.51\%, EEUU 30.98\%, Colombia 4.35\%, Brasil 4.08\%, Chile $3.53 \%$, Inglaterra 3.53\%, Argentina 2.45\%, México 1.90\%, Venezuela $1.90 \%$ e Italia 1.63\%. La tabla 1 refiere el porcentaje por país. 
Gráfico 2. Productividad diacrónica

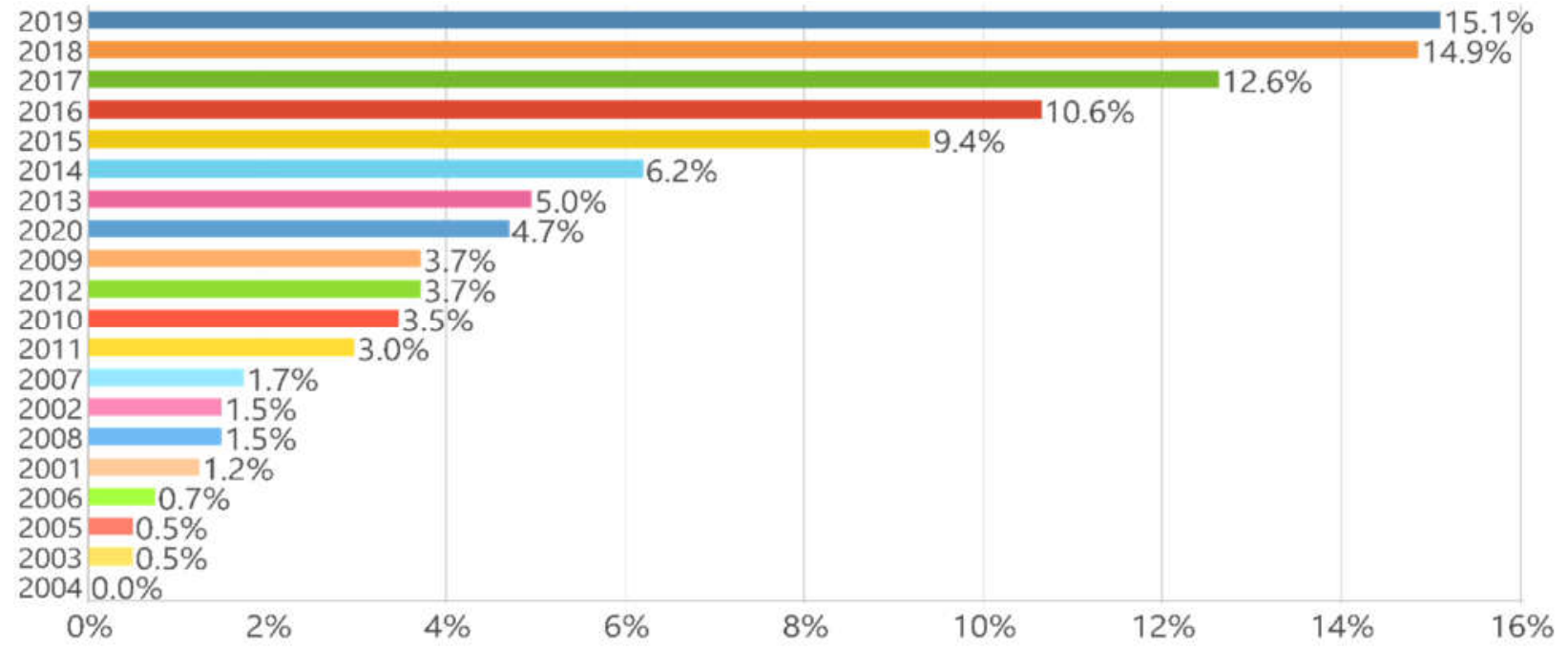

Fuente: Elaboración propia a partir de los datos procesados en MAXQDA 2020

Tabla 1. País de aplicación

\begin{tabular}{|c|c|c|c|c|c|}
\hline País & Porcentaje & País & Porcentaje & País & Porcentaje \\
\hline España & 34.51 & Malasia & 0.54 & Perú & 0.27 \\
\hline EEUU & 30.98 & Bélgica & 0.54 & Portugal & 0.27 \\
\hline Colombia & 4.35 & Cuba & 0.54 & Polonia & 0.27 \\
\hline Brasil & 4.08 & Japón & 0.54 & Puerto Rico & 0.27 \\
\hline Chile & 3.53 & Taiwán & 0.54 & Tailandia & 0.27 \\
\hline Inglaterra & 3.53 & Francia & 0.27 & Rumania & 0.27 \\
\hline Argentina & 2.45 & Finlandia & 0.27 & Eslovenia & 0.27 \\
\hline México & 1.90 & Holanda & 0.27 & Eslovaquia & 0.27 \\
\hline Venezuela & 1.90 & Austria & 0.27 & Serbia & 0.27 \\
\hline Italia & 1.63 & Chipre & 0.27 & Suecia & 0.27 \\
\hline Turquía & 1.36 & China & 0.27 & Bélgica & 0.27 \\
\hline Canadá & 1.09 & Alemania & 0.27 & & \\
\hline Australia & 0.82 & Ecuador & 0.27 & & \\
\hline
\end{tabular}

Fuente: Elaboración propia a partir de los datos procesados en MAXQDA 2020

No resulta extraño haber encontrado estudios de la enseñanza del español en países como Turquía, Eslovaquia, Rumania, Austria, Bélgica, Serbia, Japón, Taiwán, China, dado que es la tercera lengua más usada para la producción de información en internet y en especial Revista Assensus | Vol. 5 | Núm. 9 | 2020 | 
en redes sociales, después del inglés y el chino (Lobachev, 2008), lo que ha contribuido a elevar las demandas en la enseñanza de esta lengua. Al cruzar esta información con Inter Live, portal que ofrece estadísticas en tiempo real, y ver la cantidad de información que circula en el mundo, se podría entender mejor la razón por la cual cada vez más países desean aprender español (Internet Live Stats - Internet Usage y Social Media Statistics, s. f.). Aproximadamente, hay 4,673,431,161 usuarios conectados por segundo.

En lo concerniente al objeto de estudio, en esos países de aplicación, se enfatiza el abordaje basado en el desarrollo de competencias socioculturales aunadas a la educación a distancia con un enfoque comunicativo y sociocultural. Por lo que se refiere al contexto de aplicación y en consonancia con las evidencias del corpus estudiado, la tendencia se sitúa en el nivel de Educación Superior (73.4\%), siendo menos relevante la capacitación de padres para alfabetizar (0.4\%), como se puede apreciar en el gráfico 3.

Gráfico 3. Contexto de aplicación de las investigaciones revisadas

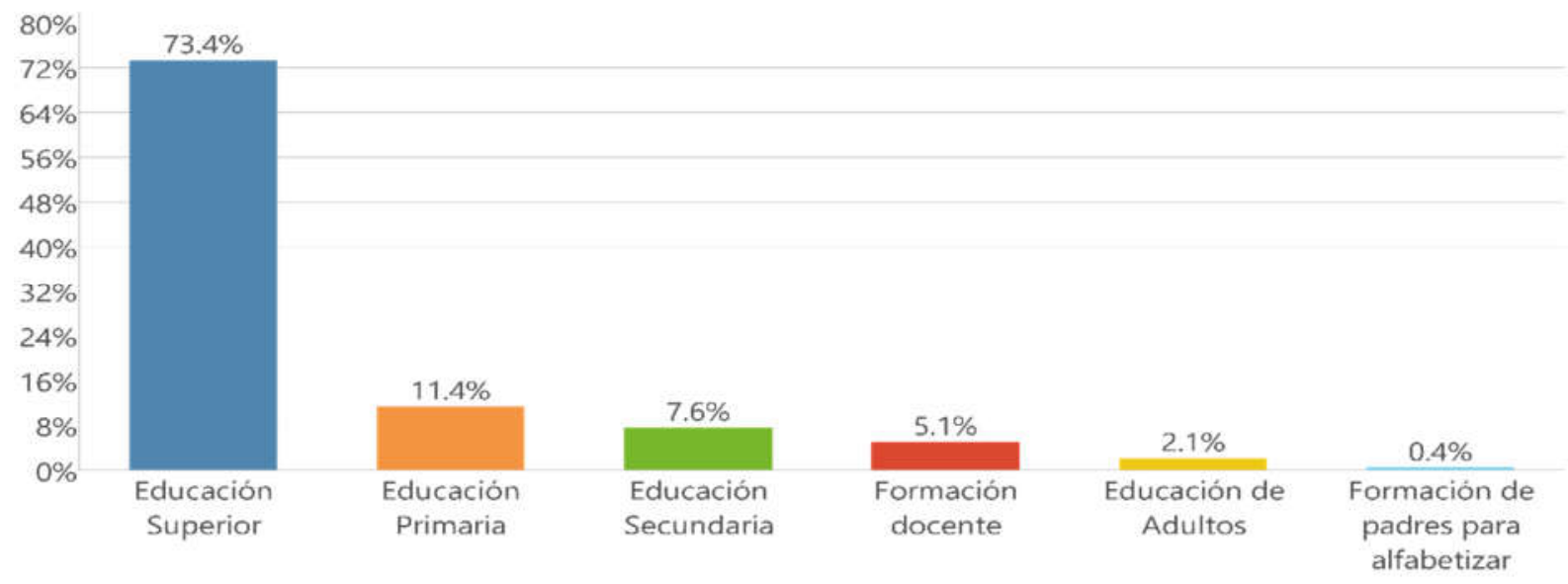

Fuente: Elaboración propia a partir de los datos procesados en MAXQDA 2020

Los resultados derivados de esta revisión sistemática se circunscriben a diversas temáticas abordadas. La supremacía recae sobre: el desarrollo de competencias, la producción oral y la escrita, el léxico (cuya totalidad de segmentos discursivos codificados se ubica en la enseñanza del español como segunda lengua), la influencia de la modalidad de la enseñanza, la escritura académica vinculada exclusivamente al nivel de Educación Superior y a la investigación, el uso de blogs y los estilos de aprendizaje basados en tarea y en proyectos. Otros tópicos vinculantes son: la consideración del género discursivo para el desarrollo de competencias, el énfasis en la necesidad de trabajo colaborativo, entre otros que se pueden visualizar en el gráfico 4.

En cuanto a las problemáticas frecuentes, se presentan entre las primeras la producción escrita (29.5\%), la competencia sociolingüística (26.9\%), la comprensión escrita (8.3\%), las competencias retóricas (7.8\%) y la lingüística de corpus para la enseñanza (6.7\%). Esta última evidencia intersección con textos modélicos, gramática pedagógica, enfoque comunicativo 
funcional, escritura académica e investigación, estrategias retóricas y conciencia metapragmática como estrategia de autorregulación. En menor medida se encuentra literatura no abordada desde lo estético, aplicación de un enfoque comunicativo en papel, coherencia y cohesión, progresión temática, uso de diminutivos, entre otros. El gráfico 5 muestra la distribución en torno a la dimensión mencionada.

Gráfico 4. Segmentos discursivos localizados por temáticas abordadas

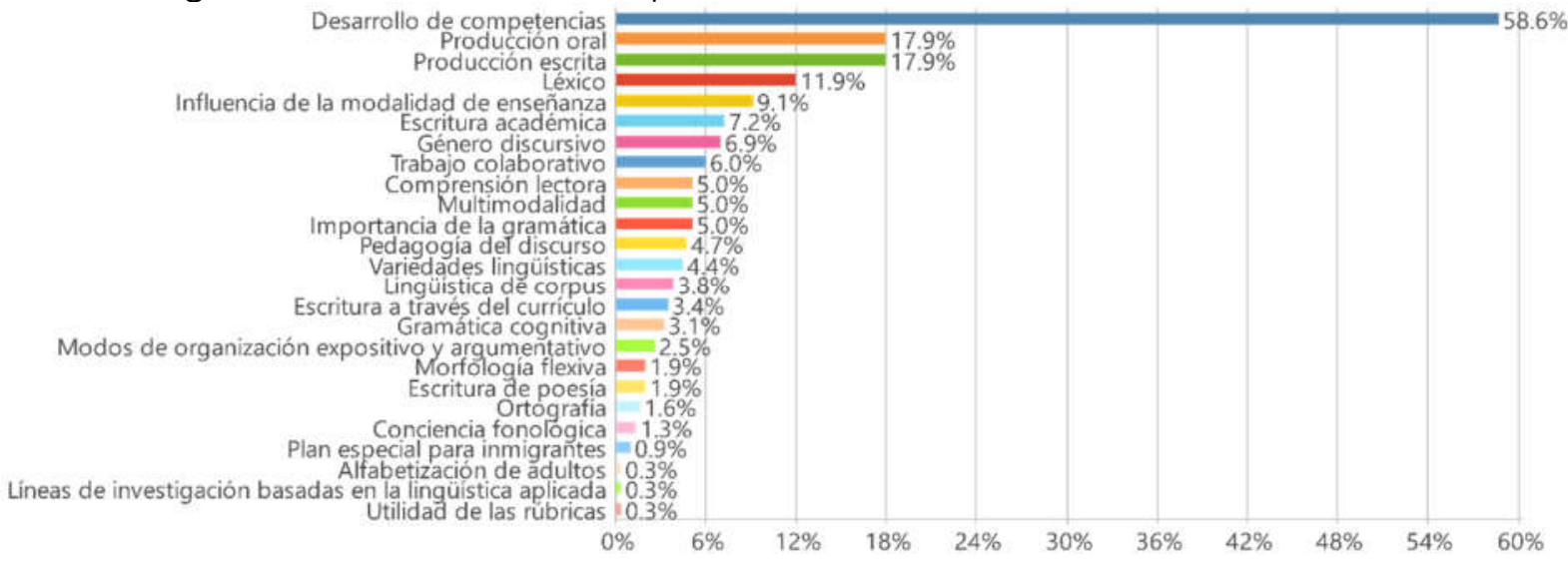

Fuente: Elaboración propia a partir de los datos procesados en MAXQDA 2020

Gráfico 5. Problemáticas frecuentes en el corpus de revisión

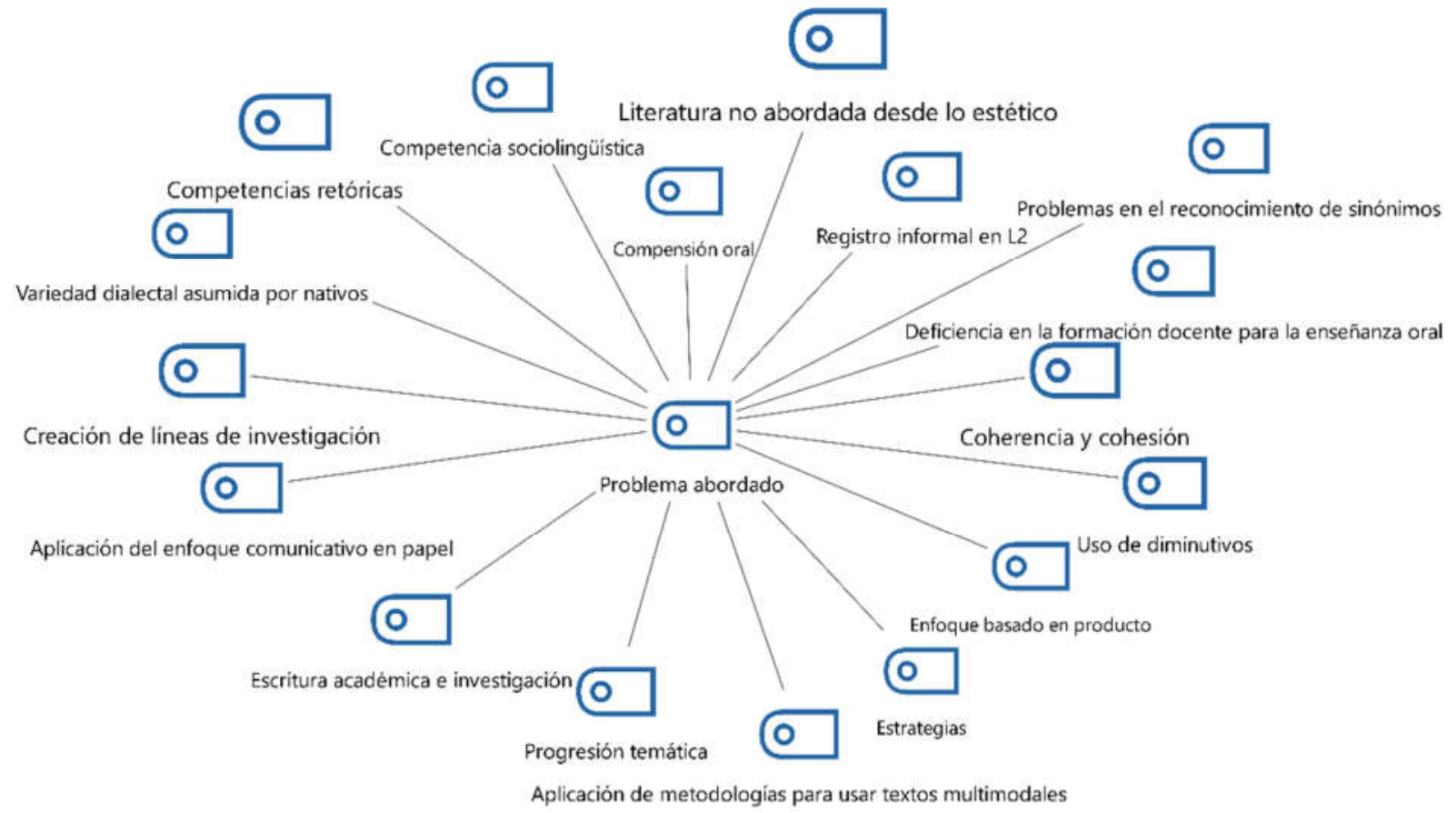

Fuente: Elaboración propia a partir de los datos procesados en MAXQDA 2020

Revista Assensus | Vol. 5 | Núm. 9 | 2020 | 
En lo concerniente a la dimensión enfoques de enseñanza y tendencias metodológicas, se presenció un mayor porcentaje en los enfoques, seguido de las metodologías basadas en estilos de aprendizaje y en menor medida, los textos modélicos, la transposición didáctica y la teoría de la valoración, lo que se evidencia en el gráfico 6.

Gráfico 6. Enfoques de enseñanza de la lengua y tendencias metodológicas

$$
\text { Metodologías basadas en estilos de aprendizajes }
$$

Autorregulación

Tendencias metodológicas que involucren la tecnología

Discurso

Juego como estrategia de aprendizaje

Lectura

Desarrollo de competencias retóricas

Secuencia didáctica

Gramática

Modalidad

Disponibilidad léxica

Textos modélicos

Transposición didáctica

Teoría de la valoración I

Fuente: Elaboración propia a partir de los datos procesados en MAXQDA 2020

Acerca de los enfoques y metodologías, se revela que en primer lugar están los enfoques de enseñanza de la lengua. Nos encontramos con una realidad intuida, como puede observarse en el gráfico 7 , y es que la mayoría de los estudios considera un enfoque comunicativo, lo cual tiene mayor sentido si discurrimos en torno a que el corpus está conformado por artículos que estudian la enseñanza del español como L1 y L2.

Gráfico 7. Tendencias en los enfoques de enseñanza de la lengua

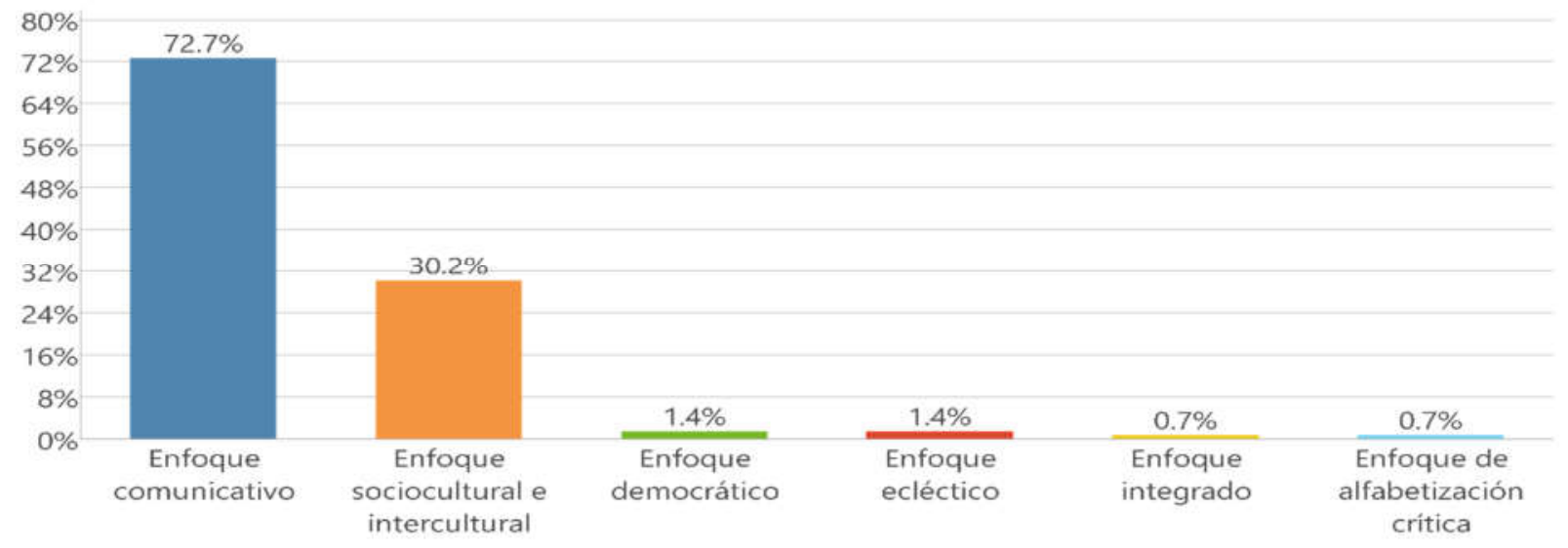

Fuente: Elaboración propia a partir de los datos procesados en MAXQDA 2020 
Luego de los enfoques, mantienen supremacía las metodologías basadas en diversos estilos de aprendizaje como se puede apreciar en la tabla 2.

Tabla 2. Metodologías basadas en estilos de aprendizajes

\begin{tabular}{ll}
\hline Estilos de aprendizajes & Porcentaje \\
\hline Aprendizaje basado en tareas & 27.43 \\
Aprendizaje colaborativo & 18.58 \\
Tutorías & 15.93 \\
Aprendizaje Integrado de Contenido y Lenguaje & 15.04 \\
Aprendizaje servicio & 9.73 \\
ABP & 7.08 \\
Aprendizaje de la lengua en comunidad CLL & 6.19 \\
\hline Total & 100.00 \\
\hline
\end{tabular}

Fuente: Elaboración propia a partir de los datos procesados en MAXQDA 2020

Seguidamente, los resultados muestran la presencia de la autorregulación al promover situaciones de aprendizaje en las que el estudiante reflexione metacognitivamente; por ejemplo, Ziegler y Moeller (2012) usaron un portafolio apoyados de la evaluación formativa. En este sentido, hallamos un $47.01 \%$ en estrategias destinadas a la toma de conciencia metapragmática, un $26.50 \%$ a la coevaluación (retroalimentación mediada), un $17.95 \%$ a estrategias cognitivas, un $6.84 \%$ a prácticas de antes, durante y después de los procesos comunicativos y un $1.71 \%$ a metodología cognitiva aplicada. Luego de lo anterior, se observa que las preferencias apuntan a tendencias metodológicas que involucran la tecnología; por supuesto que de igual manera coocurren con los enfoques y estilos de aprendizaje en gran medida, por lo que sigue manteniendo el primer lugar el enfoque comunicativo y en lo que ocupa al estilo de aprendizaje se establece una relación muy cercana con el aprendizaje colaborativo. Las herramientas más usadas en la enseñanza de la lengua se muestran en el gráfico 8:

Gráfico 8. Tendencias metodológicas que involucren la tecnología

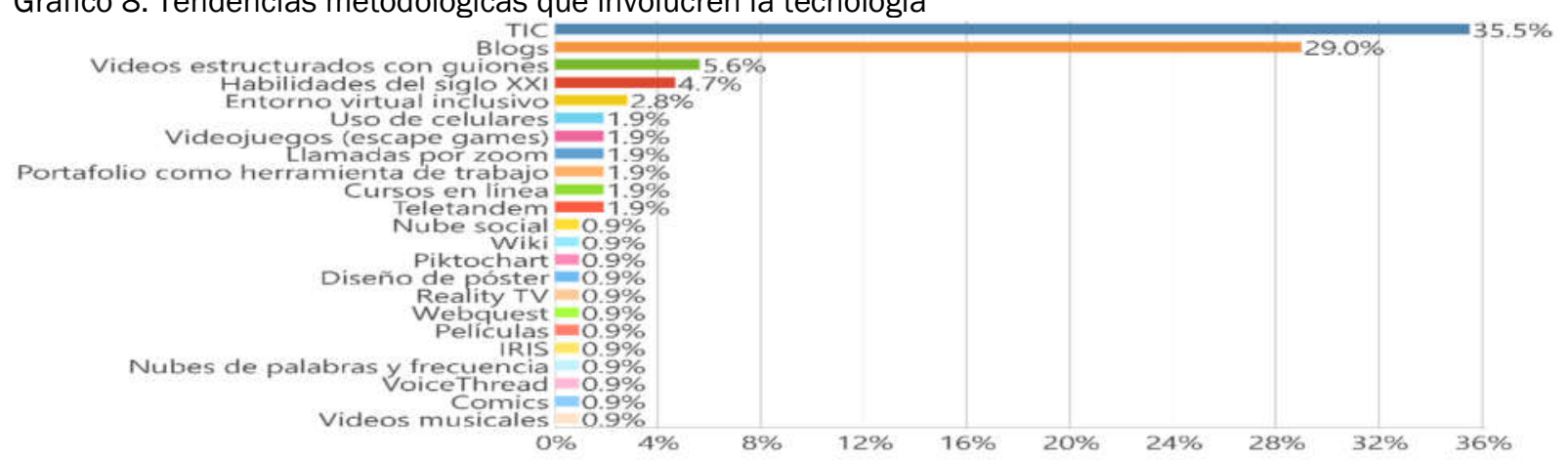

Fuente: Elaboración propia a partir de los datos procesados en MAXQDA 2020 
En lo que respecta a las tendencias del ámbito discursivo, se categorizó la pedagogía del discurso en $38.10 \%$, la multimodalidad en un $28.57 \%$, la pedagogía basada en los géneros $21.43 \%$, abordaje de la perspectiva de género $7.14 \%$, análisis del discurso y análisis crítico del discurso en $2.38 \%$ cada uno. Llama la atención la poca relevancia que se le atribuye al análisis del discurso en el marco de las investigaciones pedagógicas, en especial si cavilamos con respecto a los beneficios en el desarrollo del pensamiento crítico y a la contribución de una ciudadanía global en unos contextos cada vez más minados de exceso de información y de noticias falsas (Martínez-Lirola y Martínez-Lirola, 2019).

Desde lo inherente al juego como estrategia de enseñanza y aprendizaje, observamos tendencias en el uso de canciones $31.25 \%$, chistes $12.5 \%$, mapas semánticos $12.50 \%$, sopas de letras, criptogramas y crucigramas $12.5 \%$, escritura creativa por medio de recortes y ensamblajes $12.5 \%$, propuesta didáctica publicitaria ofreciendo un producto $6.25 \%$, juego de roles $6.25 \%$ y metáforas $6.25 \%$. En cuanto a la lectura, se emplean como estrategias vinculantes: la lectura de poesía $55.56 \%$, la lectura para la apreciación literaria $11.11 \%$, ambientes alfabetizadores $11.11 \%$, uso de bibliotecas en el aula $5.56 \%$, crítica literaria periodística $5.56 \%$, entrenamiento estratégico en lectura $5.56 \%$ y concepción simple de lectura 5.56\%. Esta última trata sobre un modelo que explica la lectura desde la oralidad (Zevallos Polo et al., 2017). El desarrollo de competencias retóricas fue empleado estratégicamente y su intersección estuvo delimitada con códigos como ver películas, videos con guiones, enfoque sociocultural e intercultural y con el aprendizaje basado en tareas.

Para abordar la gramática, la tendencia fue la gramática pedagógica 50\%, ejercicios relacionados con el análisis gramatical y la ampliación de las estructuras 38.9\% y el estilo de enseñanza extensiva 11.11\%. Con menor impacto, hubo aplicaciones de secuencias didácticas a través de las distintas fases expuestas por Camps (2003), el abordaje de aula invertida (Moranski y Henery, 2017); incluso se confirmó que la modalidad a distancia también contribuye como una metodología al facilitar un entorno virtual amigable (Ferrerira Nobre et al., 2019).

Dado que el corpus incluía artículos de enseñanza del español como segunda lengua, se halló el uso de la disponibilidad léxica como estrategia para la enseñanza de la lengua oral ubicando el léxico de los estudiantes por centros de interés. Asimismo, se codificaron estudios que abordan el uso de textos modélicos para la enseñanza de la producción oral y escrita (Björk y Blomstrand, 2006), la transposición didáctica (Álvarez Angulo, 2013) y el uso de la teoría de la valoración (Lirola, 2010), pero en un número poco considerable. 


\section{Discusión}

\section{Temas abordados en la enseñanza de la lengua en las dos últimas décadas}

La globalización y la sociedad del conocimiento han promovido un mayor auge en el desarrollo de competencias comunicativas y así también en el número elevado de prácticas de literacidad que han hecho posible los distintos escenarios y necesidades que envuelven al ser humano. En este sentido, hay una tendencia lógica para el contexto sociocultural actual que ubica el interés en el desarrollo de competencias, la cual logró dentro del corpus de estudio un total de 193 segmentos discursivos ubicados en su mayoría en el nivel de Educación Superior, cuyas intersecciones principales se dan con la producción oral, escrita, enfoque comunicativo y sociocultural. Cox y Montgomery (2019) aseguran que justamente la realidad de la globalización ha centrado el foco en la necesidad de promover situaciones de aprendizaje para el desarrollo de habilidades; por consiguiente, destacan los beneficios de las habilidades del siglo XXI. Bajo esta premisa, investigaron la intensidad del compromiso en un proyecto de español que exigía realizar y reflexionar sobre una entrevista. Detectaron que las tareas auténticas son las que desarrollan las competencias que la ciudadanía global requiere.

Por un lado, se tienen estudios como el de Aponte Montejo (2019), quien examina las implicaciones didácticas del modelo de análisis de errores en el proceso de aprendizaje. Los aciertos de esta intervención evidencian que la mejora en la competencia lingüística del estudiante es inherente a la reflexión sobre su proceso de aprendizaje, particularmente su error. Por otro lado, se encuentran estudios como los de Taguchi et al. (2016), quienes investigaron la comprensión del significado contextual como tipo de comunicación indirecta que implica la capacidad de comprender la intención implícita mediante el uso de conocimientos lingüísticos, claves contextuales y la asunción de relevancia. Como se puede evidenciar en investigaciones como la de Escagedo (2019), en Italia, se presenta la importancia de la escucha para el desarrollo de la producción oral. Además, siguiendo los presupuestos del Análisis de la Conversación, apunta a que los estilos conversacionales tienden a variar según las lenguas y culturas; por lo tanto, el nivel discursivo es eminentemente relevante (Cornieles, 2011; Cortes Bueno, 2017; E. Enkin y Correa, 2018).

Estos resultados concuerdan con los de Navarro et al. (2016), quienes indican que un gran número de las investigaciones sobre la escritura se sitúan en pregrado y prefieren las Humanidades y las Ciencias Sociales. No obstante, difieren en cuanto a que ellos plantean que en la enseñanza del español hay una tendencia en investigaciones enfocadas en la producción escrita y las que revisamos se centran en ambas, con mayor tendencia en abordar de manera explícita géneros pertenecientes a la oralidad. Quizás, los estudios orientados a la enseñanza del español como segunda lengua sean la razón por la que en este corpus se permean más géneros discursivos de la oralidad que de la escritura. Con respecto a los países 
que han publicado un número elevado de estudios sobre el desarrollo de las competencias, se pudo evidenciar que España y EEUU son los pioneros y que desde el 2001 han sido temas constantes de estudio (Gutiérrez Arvizu, 2020; F. Harper y Hamer, 2006; Hurd et al., 2001; Level Hernández, 2009; López-García-Torres y Saneleuterio, 2020; Pérez Niño, 2010; RuizFunes, 2001; Tolchinsky et al., 2002).

Aunado a lo anterior, hay una presencia del género discursivo desde la perspectiva bajtiniana. Los estudios revisados hacen un abordaje más hacia el género primario, la conversación (Martínez, 2016). El género se materializa a través de formas típicas de organización que dan cuenta de una situación de enunciación específica propia de una esfera de la actividad social. En el caso de Krepel y Sinclair (2019), demostraron que los estudiantes se motivan en el estudio de la lengua cuando son capaces de relacionar su estudio con sus propios intereses. De este modo, el desarrollo de la producción oral se ha abordado desde actividades auténticas y espontáneas que van más allá de las planificadas en el aula. Desde otro punto de vista, Espinosa Guerri (2019) presentó un estudio de la toma de turnos como uno de los componentes necesarios para el desarrollo de la competencia pragmática, dado que generalmente en el estudio de las lenguas no es frecuente ese contenido que, a su vez, es transversal a otros.

Otro tema que llamó la atención dentro de esta dimensión fue el programa de televisión en la docencia para abordar varios aspectos de la competencia comunicativa, entre ellos: gramatical, léxico, sociocultural, pragmático, estratégico y discursivo. Destaca Del Valle Revuelta (2018) que a partir del uso de un documento audiovisual se trabajan mucho mejor los aspectos socioculturales. En este hilo temático, Ramos-González y Rico-Martín (2014) demuestran cómo han estudiado aspectos propios de la cortesía verbal a fin de desarrollar competencias lingüísticas y pragmáticas a través de actividades centradas en una serie de televisión difundida en internet. Este tipo de estudios se podrían transponer a contextos específicos del español como lengua materna para estudiar aspectos sociolingüísticos como variedades dialectales.

La producción escrita se abordó en coocurrencia con la escritura académica e investigación, comprensión escrita, pedagogía basada en los géneros y desarrollo de secuencias didácticas. El artículo de Angelo y Menegassi (2016) aborda el proceso de producción escrita del género textual respuesta basados en los fundamentos de Bajtín y las contribuciones de la Lingüística Aplicada en relación con la lectura y la escritura. Otros artículos como los de Caro Valverde et al. (2018), en los que se estudia la percepción de los docentes mientras enseñan la argumentación, muestran que en los casos de los comentarios argumentativos hay una preferencia por la retroalimentación escrita, dado que proporciona un registro individualizado de errores para un análisis más profundo y la evaluación discursiva. Incluso siguen las bases teóricas bajtinianas y abordan un nivel discursivo a través de las pautas y rúbricas. La variedad de instrumentos de evaluación es un elemento mediador en la enseñanza y el aprendizaje de una lengua, dado que se convierten en protocolos que guían el 
proceso escritural a la vez que las formas de participación, como la autoevaluación y la coevaluación. De esta manera, promueven un andamiaje de saberes y revelan la utilidad de analizar el canon o características prototípicas para fomentar el pensamiento crítico y la alfabetización en el aula.

El léxico y la gramática también han sido temas abordados, aunque el primero lo ha sido en mayor medida. El léxico registra intereses basados en incrementar la comprensión lectora en L2, dado que hay muchas investigaciones que han corroborado el impacto positivo de las oportunidades de recuperación de palabras para el aprendizaje intencional y contextual y no únicamente por significado (Barcroft, 2015). También se ha presentado dentro de este ámbito la disponibilidad léxica como metodología para identificar el léxico de grupos de aprendices de español como L2 (Martín y Cabrera, 2018). Asimismo, encontramos estudios dirigidos a la enseñanza de vocabulario para el desarrollo de la velocidad y automaticidad del acceso al léxico bajo un enfoque incidental y explícito, los cuales conducen en conjunto a una mejora significativa en la velocidad y automaticidad del acceso al léxico de los alumnos (PellicerSánchez, 2015).

En cuanto a la gramática, no fue un tema privilegiado, pero al menos conseguimos trabajos que estudian la competencia gramatical desde distintos ámbitos, por ejemplo, la enseñanza de las funciones gramaticales del se (López Quero, 2017). Desde el enfoque funcional encontramos diseños de propuestas para el proceso de enseñanza del verbo centrado en la capacidad comunicativa (lingüística, metalingüística, pragmática y discursiva) a través de actividades creativas (Monarca y Llancao, 2016). Otros apuntan hacia el valor sociolingüístico e indican que en el caso del español L1 es infrecuente que se estudie esta información, la cual va más allá de atender a diferencias dialectales o de registro; más bien la insistencia es de incorporar la información sociolingüística en la didáctica de una lengua (Rodrigues, 2019; Terradez Gurrea, 2017). Cerramos con la gramática desde una perspectiva que concierne a los educadores, y es la importancia de la gramática cognitiva para la enseñanza del español, dada la relevancia que tiene entender el funcionamiento de las estructuras del lenguaje y provocar procesos metalingüísticos en los estudiantes (Bosque y Gallego, 2016; Estrada Chichón y Zayas Martínez, 2019; Simon, 2018).

La escritura académica estuvo presente como tema y desde la transversalidad, acompañada de la relevancia del habla pública en escritura de los edublogs, considerados como un fructífero escenario para la construcción colaborativa del conocimiento. A su vez, sugiere una tendencia a involucrar distintas áreas para el desarrollo de las habilidades productivas (Hernández López, 2018). Se constató la exaltación del ambiente colaborativo, la incorporación de la investigación, el desarrollo de competencias retóricas y la trascendencia de la escritura a través del currículo como parte importante en la enseñanza del español. Distintos estudios han demostrado entre sus resultados una predisposición positiva para el desarrollo de habilidades cognitivas complejas (Bocanegra-Valle y Basturkmen, 2019; Campillo-Ferrer et al., 2020; Lyster y Ballinger, 2011; Santos, 2019; Serafini y Torres, 2015). 
Bien sea en el desarrollo del discurso oral o escrito, dentro del ámbito académico o literario, se presentaron temas que privilegian la multimodalidad discursiva, abarcados desde la sociosemiótica y la integración del análisis del discurso, competencia sociolingüística e intercultural y la alfabetización académica (Escobar Urmeneta, 2013; Farías et al., 2007; Farías y Véliz, 2019; Lee, 2014; Nikleva, 2018; Ramos-González y Rico-Martín, 2014; Verdessi Hoy, 2015). También, para este tópico hay estudios que revelaron la relación de una evaluación formativa a través de la retroalimentación agregando screencasts personalizados para elevar la calidad de las producciones, aprovechando la pedagogía del error y superando las brechas de una retroalimentación tradicional escrita (Elola y Oskoz, 2016; Felicity Harper et al., 2018).

Dentro de la línea temática, ha sido un tópico colateral el trabajo colaborativo, el cual se vincula a menudo con las tecnologías de información y comunicación ( Hernández López, 2018; Lee, 2014; Morán López, 2016; Pomata García y Diaz Ayuga, 2017). El trabajo colaborativo amerita una detallada secuenciación de las tareas para elevar el compromiso cognitivo de los estudiantes y el desarrollo del lenguaje en contextos educativos. Además de la estructuración de las tareas, es relevante que las mismas pertenezcan a las demandas comunicativas del mundo real, es decir, enmarcarlas en situaciones auténticas (GurzynskiWeiss, 2016b).

Como se ha destacado, los temas relacionados con la enseñanza de la lengua hacen énfasis en el aprendizaje basado en tareas (Isuka lizuka, 2019) y en casi todos los estudios se nota una tendencia a enfatizar el componente social y el dinamismo de la enseñanza de la lengua desde el conectivismo, la ubicuidad y la influencia positiva de una modalidad a distancia o semipresencial (Dona et al., 2014; Elizabeth Enkin y Mejias-Bikandi, 2017; LópezGarcía-Torres y Saneleuterio, 2020; Russell y Curtis, 2013; Sedano Cuevas, 2017).

\section{Problemas abordados en el corpus de revisión}

Actualmente, la enseñanza de la lengua posee un alto grado de relevancia, no solo por su rigor científico y su amplio campo de acción, sino por su teleología (del griego té

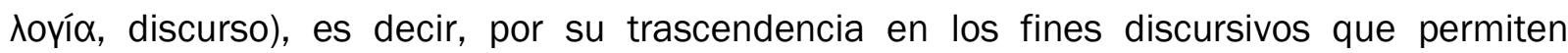
desarrollar la interacción con el mundo y asirse del conocimiento. Así, se considera la trayectoria que se va dando en torno a los distintos niveles del lenguaje, desde el menos complejo, el ejecutivo, hasta llegar al más complejo, el epistémico. De esta manera, se pretende formar a un individuo más crítico, que sea capaz de mostrar su propia voz en su discurso oral y escrito e interpretar información de distintas fuentes y destinatarios. En el corpus de estudio, se presenta de manera recurrente la problemática en producción escrita relacionada con una multiplicidad de variantes que analizamos desde distintos marcos. En el marco lingüístico, aparece involucrada con dificultades desde las propiedades del texto como lo son la coherencia y la cohesión (Barrigüete, 2010; Benavides, 2015; Gutiérrez Arvizu, 2020; 
Taguchi et al., 2016) y también desde la comprensión escrita (Clares y Morga, 2019; T. Martínez et al., 2009; Zevallos Polo et al., 2017). Es relevante mencionar que la comprensión oral se computó dentro de las limitaciones que ameritan estudio y que ha sido metodológicamente abarcada a partir de videos estructurados con preguntas y comentarios, tutorías y por medio del CLL (Ambard y Ambard, 2012; Azorin Abellan, 2019; Del Valle Revuelta, 2018).

Otro problema recurrente se circunscribe al marco sociolingüístico. Se estudia la competencia sociolingüística desde el aprendizaje de una lengua materna o de una segunda asociada a la competencia comunicativa intercultural crítica (López-Jiménez, 2018; Terradez Gurrea, 2017), aunada a las variedades lingüísticas y al uso apropiado del registro en ámbitos académicos e investigativos (Andion Herrero, 2019; Baralt et al., 2011; Borrego Nieto et al., 2019; Buyse, 2014). De igual modo, se considera importante mencionar que este marco vincula el desarrollo de competencias retóricas en la oralidad y en la escritura reflejado en estudios como los de Anderson y Cuesta-Medina (2019), Buyse (2014), Cambronero (2013) y Tecedor (2016).

En el marco literario, se encuentran un número reducido de estudios sobre la literatura no abordada desde lo estético, sino más bien con fines pedagógicos en los que se comentan los riesgos de enfatizar en objetivos instrumentales, tales como la adquisición de vocabulario y gramática o conocimiento cultural (Alvstad y Castro, 2009). Otros estudios conciben la literatura como apoyo al problema del desarrollo de la competencia transcultural (YáñezPrieto, 2014). El último marco, el psicopedagógico, se puede resumir en tres aspectos. El primero constata que la formación del profesorado se debe orientar a perspectivas como la disciplinaria, crítica y pragmática (Adlerstein et al., 2016). El segundo es trascendental para la temática de la aplicación del enfoque comunicativo, pues en muchos casos se da en papel (Level Hernández, 2009); es decir, en las revisiones exhaustivas en torno a metodologías aplicadas se suele evidenciar otro estilo de enseñanza. No obstante, está la posibilidad de su ejecución obviando aspectos gramaticales o basándose en actividades pseudocomunicativas y dejando a un lado los postulados de la lingüística textual, parte de las bases teóricas que sustentan el enfoque (Casado Velarde, 1993, 2000; Wingate, 2018). El tercero apunta a la necesidad de fundamentar las prácticas pedagógicas en una psicología cognitiva, lo cual implicaría un traslado de un enfoque basado en productos hacia la promoción del proceso a través del uso de estrategias cognitivas y metacognitivas (De Zarobe y Zenotz, 2015).

\section{Enfoques y tendencias metodológicas}

El enfoque comunicativo constituye un área muy estudiada en lo que respecta a las tendencias metodológicas en la enseñanza de las lenguas extranjeras, tal como lo afirma Santos Díaz (2017). De hecho, sus principios son transferibles a la enseñanza de la lengua materna (Canales, 2010), cuyo propósito fundamental es desarrollar la competencia 
comunicativa de los estudiantes (Ramírez Guerrero et al., 2019). Este enfoque es tan amplio que se evidencia en los fundamentos curriculares de múltiples países, como por ejemplo de los 19 que formaron parte del estudio ERCE 2019.

Resulta interesante resaltar que el enfoque comunicativo aparece contrastado con enfoques tradicionales que aún prevalecen en la práctica y en los libros de texto (Ashoori Tootkaboni, 2019; Gurzynski-Weiss et al., 2015), con el enfoque basado en contenidos (Tedick y Young, 2018) y con el enfoque crítico literario (Valério y Mattos, 2018). A propósito de esto último, una de las carencias señaladas por Gónzalez Cobas (2019) es el olvido del uso de los textos literarios en las clases de lengua. Sin embargo, junto a las tendencias metodológicas que apuntan a la lectura, aparecieron trabajos que abordan el uso de textos narrativos literarios (Rojas Moreno, 2019), la crítica literaria (Bea y Gonzalo, 2014), el uso didáctico de la poesía (Galván, 2006; Lanseros Sánchez y Sánchez García, 2017, 2018; Martínez Canton y Ruiz Fabo, 2019) y la apreciación literaria (García-Dussán, 2016).

Algunos artículos analizados corroboran que el enfoque comunicativo integra lo textual y lo funcional, pues toma en cuenta los distintos géneros discursivos y el uso real de la lengua (Cortes Bueno, 2017; Tolchinsky et al., 2002; Torres-Guzmán, 2011). Además, que este busca el desarrollo de las habilidades comunicativas con especial énfasis en las habilidades orales de escuchar y hablar (Cornieles, 2011; Gutiérrez Ospina y Fernan, 2020; Hernández, 2018). Empero, las percepciones de los docentes parecen apuntar a una mayor necesidad de práctica y acompañamiento de las habilidades orales (Plo et al., 2014).

En el contexto de la enseñanza de lenguas extranjeras, el enfoque comunicativo dialoga con el enfoque humanista (Marchi y Las, 2013), con el enfoque por tareas (Taourite y RuizCecilia, 2020; Villalba Ibánez, 2019) y, con mayor amplitud, con el enfoque sociocultural (Lacorte, 2007) que pone en contacto al usuario con experiencias reales de comunicación y con la cultura de la sociedad donde se inserta para aprender su lengua de destino. En tal sentido, el enfoque comunicativo converge, en primer lugar, con las tendencias metodológicas que aluden a la modalidad de aprendizaje de lenguas extranjeras, tales como la telecolaboración (Lenkaitis et al., 2019) y la inmersión total (López-García, 2017). En segundo lugar, con las metodologías que incorporan la tecnología (Hernández, 2018), por ejemplo, el uso de edublogs, canciones y videos musicales, y en tercer lugar, con aquellas que contemplan el uso de textos modélicos, como mitos, leyendas y refranes (Rojas Moreno, 2019).

Al tratarse de publicaciones sobre tendencias y enfoques metodológicos en la didáctica de la lengua, es inevitable que otro punto de interés sea encontrar la variable docente. En ese orden, el estudio de Garrote et al. (2018) revela que las prácticas de los profesores de lenguas extranjeras en el nivel secundario están claramente orientadas al enfoque comunicativo. También aparecen conclusiones interesantes que impactan los procesos acreditativos de los docentes en cuanto a su formación en didáctica de la lengua de acuerdo con la perspectiva comunicativa (Khong et al., 2018; Kissau y Algozzine, 2017; Swanson, 2017). Una de ellas 
apunta a la necesidad de ofrecer, mediante los planes de estudio, estrategias de enseñanza efectivas y específicas para desarrollar habilidades lingüísticas, más allá de formar a los futuros docentes en lo que concierne únicamente a corrientes pedagógicas generales (Pizarro, 2013). Asociados a estos hallazgos, se encuentran en menor medida aportes que se refieren a la transposición didáctica, tanto en relación con el aprovechamiento de los textos para elaborar propuestas didácticas (Rodríguez Mata, 2019), como con la necesidad de armonizar la teoría con la práctica (Ainciburu y Rodríguez, 2014; Lantolf, 2011).

En el corpus objeto de estudio, apenas se evidencia la secuencia didáctica (SD) como tendencia metodológica que propone el desarrollo de la comprensión lectora y de la competencia léxica (Sanjuan Álvarez y del Moral Barriguete, 2019), lo cual podría estar indicando que es preciso generar conocimiento acerca de las implicaciones que tiene su implementación para el desarrollo de las habilidades comunicativas, del vocabulario y el aprendizaje de la gramática funcional. La poca aparición de la SD en el corpus estudiado podría explicarse porque los requerimientos metodológicos de las publicaciones científicas dificultan reportar su aplicación, dado que privilegian el carácter empirista.

Llama a la reflexión que en una sociedad en cuyos marcos curriculares se promociona una ciudadanía crítica, apenas existan publicaciones centradas en investigar el enfoque democrático (Benedet, 2009; Remesal y Brown, 2015), el enfoque integrado (McMullin, 2016) y el enfoque de alfabetización crítica (Valério y Mattos, 2018; Villalba Ibánez, 2019). Si buscamos que los usuarios de la lengua adquieran aprendizajes para la vida y sean críticos al momento de comprender y producir, debería existir mayor interés en indagar si la metodología de enseñanza utilizada en las aulas realmente promueve esas competencias o si nos estamos quedando en meras intenciones y propuestas. Estos hallazgos coocurren con el bajo porcentaje de trabajos que abordan la transposición didáctica.

En la revisión realizada se detectaron 107 segmentos codificados en relación con el uso de la tecnología, la cual se ha convertido en una aliada para apoyar los procesos de enseñanza-aprendizaje de todas las áreas y la didáctica de la lengua no es la excepción. En los artículos objeto de estudio aparecieron recursos tecnológicos para desarrollar la competencia comunicativa, como la gamificación (Rachels y Rockinson-Szapkiw, 2018), redes sociales (Piriyasurawong, 2019), herramientas virtuales adaptadas al contexto académico (Rodrigo, 2017), edublogs (Hernández, 2018), uso de películas (Hertel y Harrington, 2015), videos musicales (Sánchez-Vizcaíno y Mora, 2018), canciones (Hornillos y Roa, 2015), diseño de telediarios (Hernández Liechti, 2017) y otros proyectos. Sin embargo, Ilama la atención lo que sostienen (González Martínez et al., 2015) en una de las conclusiones de su estudio: no siempre la tecnología empleada contribuye con la mejora de las habilidades textuales.

Con el auge de la modalidad de educación a distancia producto de la crisis sanitaria actual (COVID-19), la incorporación de la tecnología se convierte en un punto de interés. En lo que concierne a la didáctica de la lengua, es necesario saber encauzar su uso, de modo que las actividades que se diseñen desarrollen efectivamente las competencias comprensivas y 
productivas de los estudiantes, tanto en el plano oral como en el escrito. Los ejemplos mencionados más arriba aparecieron como tendencias empleadas en la enseñanza de lenguas extranjeras en mayor medida que en la lengua materna. Por tanto, sería fundamental plantearnos la siguiente pregunta: ¿Por qué no se incorporan estas herramientas en las prácticas de enseñanza de la lengua materna en la misma proporción?.

En cuanto a las tendencias metodológicas relacionadas con la autorregulación, el desarrollo de la competencia pragmática se identificó en múltiples artículos que convergen a su vez con tendencias referentes a las competencias retóricas y al discurso. Las mismas están centradas en que el sujeto que aprende pueda desenvolverse a través de textos completos que se ajusten a destinatarios, intenciones y situaciones reales de comunicación (De PablosOrtega, 2016; Rodríguez-Gonzalo y Zayas, 2017; Secchi, 2017; Sessarego, 2016). Ello supone priorizar el uso de textos auténticos por encima de textos creados con fines estrictamente pedagógicos (Yepes y Krishnamurthy, 2010). Asimismo, resulta alentador el abordaje de la competencia léxica y gramatical (Loewen et al., 2020; M. N. R. Rodríguez y García-Perera, 2016), las cuales se presentan al servicio de la comunicación, en contraposición con los enfoques tradicionales que abogaban por una enseñanza prescriptiva y estructuralista de la lengua.

Cabe destacar que las tendencias metodológicas para potenciar el léxico aparecen estudiadas desde el punto de vista de la producción oral, los libros de texto y otros recursos lexicográficos. Dicho campo podría abrir sus posibilidades al desarrollo del vocabulario en las demás destrezas: comprensión oral, comprensión y producción escritas. En consecuencia, todos estos hallazgos podrían encontrar sentido en un enfoque pragmático-discursivo. ¿Para qué deben los estudiantes aprender lengua si no es para comunicarse en un contexto, con unos destinatarios y unos propósitos concretos, a la vez que se construyen como sujetos discursivos?.

Otros puntos de acceso a la comprensión de la autorregulación como abordaje metodológico y que a la vez dan sentido a su puesta en ejecución lo ofrecen: la conciencia metapragmática (Showstack, 2016), el aprendizaje autónomo (Hurd et al., 2001), las emociones del sujeto que aprende (Sánchez-Vizcaíno y Mora, 2018), la capacidad reflexiva y de pensamiento crítico (Pérez-Valverde y Ruiz, 2014), la retroalimentación mediada por parte del docente (Gurzynski-Weiss, 2016a), la autoevaluación y las estrategias metacognitivas (Pérez-Valverde y Ruiz, 2012), la transferencia interlingüística de las habilidades de L1 a L2 (Sparks et al., 2019) y los ejercicios de automonitoreo (Thompson, 2012). No obstante, como afirman Van Compernolle et al. (2016), hace falta estudiar más cómo se aborda en la clase de lengua la reflexión sobre el desempeño gramatical, más allá de los conocimientos conceptuales. 


\section{Conclusiones}

Del corpus recabado en enseñanza del español, conformado por 404 artículos en el rango de 2001-2020, quedaron 290 artículos de Scopus, 83 de Web of Science y 31 de SciELO. El corpus demostró que la productividad diacrónica responde a la ley de Price (1986), pues el aumento en la producción científica ha sido significativamente ascendente en la última década a diferencia de la anterior. España y EEUU han sido pioneros en la publicación de artículos en el área, con un marcado énfasis en el contexto de la Educación Superior. América Latina no tuvo la suficiente presencia, por lo que se sugiere una nueva revisión en otras bases de datos a fin de darle mayor visibilidad a las investigaciones de la enseñanza del español como lengua materna en el ámbito latinoamericano, dado que en su mayoría se circunscribieron a la enseñanza del español como segunda lengua.

Los principales objetos de estudio, problemáticas abordadas, tendencias y enfoques metodológicos empleados en la enseñanza de la lengua española se presentan integrados en cuatro marcos: el lingüístico, el sociocultural, el literario y el psicopedagógico. Por lo que se refiere al marco lingüístico, hubo un alto nivel de consistencia entre objeto de estudio, problemática y enfoques metodológicos centrados en el desarrollo de la competencia comunicativa. Asimismo, se reflejó una consistencia en el bajo porcentaje que obtuvieron los estudios enfocados en el ámbito discursivo. En tal sentido, es mucho lo que todavía se puede recorrer en el camino de la investigación en pedagogía del discurso, principalmente en lo que respecta a los géneros, al análisis del discurso y al análisis crítico del discurso. Todo ello con el fin de contribuir con un aprendizaje de la lengua más allá de su carácter instrumental, ya que el locutor/interlocutor no es solo un sujeto empírico, sino un sujeto discursivo que, a través de la lengua, construye conocimiento (función epistémica) y configura su propia comprensión del mundo que le rodea. Esto adquiere mucho más sentido al corroborar que los estudios en enseñanza de la lengua del corpus seleccionado se situaron en el contexto de la Educación Superior.

En lo concerniente al marco sociocultural, hubo una relación entre la proporción en que se aborda el enfoque sociocultural, las variedades lingüísticas y el léxico como objetos de estudio y las problemáticas de la competencia sociolingüística, las variedades dialectales asumidas por nativos y el registro informal en lenguas extranjeras. Cabe destacar que, para promover un usuario de la lengua con dominio de las competencias socioculturales, la tendencia se enmarcó en involucrar constantemente las TIC. Es preciso señalar que los estudios dirigidos a la perspectiva de género e inclusión estuvieron casi ausentes. Desde lo literario, se identificó como tema la escritura de poesía; como problema hallamos la literatura no abordada desde lo estético y como tendencia metodológica encontramos la lectura, cuya vertiente literaria se puso de manifiesto en el uso de textos narrativos y poéticos, bibliotecas, apreciación y crítica literaria. No obstante, este marco carece de la multiplicidad de opciones 
que ofrece la literatura para el desarrollo de la competencia literaria como parte de la competencia comunicativa. Pudimos constatar un distanciamiento del valor estético y artístico, dado que el énfasis estuvo dirigido hacia la adquisición de habilidades socioculturales.

En cuanto al marco psicopedagógico, los temas estuvieron en sintonía con teorías constructivistas, especialmente a partir de la interacción social vygotskyana. Igualmente, se expusieron temas como la relevancia positiva de la modalidad virtual o mixta, metodologías basadas en estilos de aprendizaje con mayor énfasis en la autorregulación de los procesos cognitivos, conciencia metapragmática y las tendencias que involucran herramientas tecnológicas. Entre los problemas se destacaron la aplicación del enfoque comunicativo en papel y deficiencia en la formación docente para el desarrollo de las habilidades orales. En el caso del español como segunda lengua, surgió reiteradamente la coevaluación para mediar y realizar la retroalimentación. Generalmente, se aplicó en su mayoría por parte del docente y no entre pares académicos, cuya intención fue la de fomentar la reflexión en los estudiantes en cuanto a los errores cometidos. Es importante dejar claro que la enseñanza de la lengua desde cualquier enfoque, estilo de aprendizaje o tendencia metodológica debe orientarse a empoderar a los estudiantes e ir más hacia el nivel discursivo, lo que permitirá el desarrollo de competencias cognitivas, emocionales, comunicativas e integradoras requeridas para interactuar como sujeto democrático y pluralista en una sociedad (Rodríguez y Hernández, 2014).

Entonces, ¿en qué se necesita fortalecer la investigación en didáctica de la lengua española? Se requiere estudiar la enseñanza de la lengua considerando también competencias informacionales, educación mediática, formación para resolver problemas del mundo real, desarrollo del pensamiento crítico y divergente, reflexión sobre la validez de la información en la sociedad del conocimiento. Además, que analice los discursos y que comprenda sus encadenamientos y encantamientos y así favorecer la configuración de una ciudadanía global que abre un nuevo marco al promover paralelamente una (re) alfabetización funcional, tecnológica e informacional desde la transversalidad. Asimismo, la coyuntura de la contingencia sanitaria conduce a fortalecer, más todavía, el aprendizaje autónomo para favorecer competencias ciudadanas (UNESCO, 2020).

En fin, se necesita que las investigaciones orienten el quehacer educativo para que el sujeto aprenda a producir una diversidad de géneros discursivos y a leer el mundo en una cultura multivariada y multimodal. 


\section{Referencias}

Adlerstein, C., Pardo, M., Díaz, C., y Villalón, M. (2016). Formación para la enseñanza del lenguaje oral y escrito en carreras de educación parvularia: Variedad de aproximaciones y similares dilemas. Estudios pedagógicos (Valdivia), 42(1), 17-36. https://doi.org/10.4067/S071807052016000100002

Ainciburu, M. C., y Rodríguez, M. L. R. (2014). Synonymy and processing load. A lexical decision task for natives and non-natives of neighboring languages,cognates, neighboring languages, lexical processing, teaching ELE. Calidoscopio, 12(3), 356-366. https://doi.org/10.4013/cld.2014.123.10

Álvarez Angulo, T. (1998). Las ciencias del lenguaje y su transposición en el marco de la Didáctica de la Lengua. Didáctica. Lengua y Literatura, 10, 179-188.

Alvstad, C., y Castro, A. (2009). Conceptions of literature in university language courses. Modern Language Journal, 93(2), 170-184. https://doi.org/10.1111/j.1540-4781.2009.00854.x

Ambard, P. D., y Ambard, L. K. (2012). Effects of Narrative Script Advance Organizer Strategies Used to Introduce Video in the Foreign Language Classroom. Foreign Language Annals, 45(2), 203-228. https://doi.org/10.1111/j.1944-9720.2012.01189.x

Andion Herrero, M. A. (2019). From Lectocentrism to Plurinormativism. Reflections on the Variety of Spanish in Teaching as a Second or Foreign Language. Estudios Filologicos, 64, 129-148. https://doi.org/10.4067/s0071-17132019000200129

Aponte Montejo, C. (2019). Análisis de errores y retroalimentaciones correctivas en interacciones español-portugués mediante el contexto teletándem. Íkala, Revista de Lenguaje y Cultura, 24(3). https://doi.org/10.17533/udea.ikala.v24n03a07

Arnáez Muga, P. (2006). La lingüística aplicada a la enseñanza de la lengua: Una línea de investigación. Letras, 48(73), 349-363.

Ashoori Tootkaboni, A. (2019). Teachers' beliefs and practices towards communicative language teaching in the expanding circle. Revista Signos, 52(100), 265-289.

Azorin Abellan, C. (2019). Designing and evaluating a plan to attend to immigrant students with no knowledge of spanish. Contextos Educativos-Revista de Educación, 23, 179-197. https://doi.org/10.18172/con.3487

Baralt, M., Pennestri, S., y Selvandin, M. (2011). Action research: Using wordles to teach foreign language writing. Language Learning and Technology, 15(2), 12-22.

Barcroft, J. (2015). Can Retrieval Opportunities Increase Vocabulary Learning During Reading? Foreign Language Annals, 48(2), 236-249. https://doi.org/10.1111/flan.12139

Barrigüete, C. M. (2010). How to teach Spanish as a second language based on a textual progression method. Porta Linguarum, 13, 89-101.

Bea, A., y Gonzalo, C. R. (2014). Journal's literary criticism as a didactic tool in the Faculty of Education. Cultura, Lenguaje y Representacion, 13, 51-68. https://doi.org/10.6035/clr.2014.13.3 
Benavides, C. (2015). Using a Corpus in a 300-Level Spanish Grammar Course. Foreign Language Annals, 48(2), 218-235. https://doi.org/10.1111/flan.12136

Benedet, S. M. (2009). The demystification of knowledge acquisition: Skills based approach to teaching literature. International Journal of Learning, 16(8), 441-446.

Björk, L., y Blomstrand, I. (2006). La escritura en la enseñanza secundaria: Los procesos del pensar y del escribir (4ta.). Graó.

Bocanegra-Valle, A., y Basturkmen, H. (2019). Investigating the teacher education needs of experienced ESP teachers in Spanish universities. Ibérica, 2019(38), 127-150.

Bolívar, A. (2020). La escritura de un buen artículo científico en Educación y el entrenamiento de profesores universitarios en el discurso académico. Paradigma, 41, 222-250.

Borrego Nieto, J., Recio Diego, Á., y Tomé Cornejo, C. (2019). Dos aportaciones de la NGLE a la enseñanza del español LE/L2: Panhispanismo y descripción gramatical. Journal of Spanish Language Teaching, 6(2), 217-231. https://doi.org/10.1080/23247797.2019.1676986

Bosque, I., y Gallego, Á. J. (2016). Grammar application in the classroom. Old and new teaching resources to teach grammar. RLA, 54(2), 63-83. https://doi.org/10.4067/s071848832016000200004

Buyse, K. (2014). Una hoja de ruta para integrar las tic en el desarrollo de la expresión escrita: Recursos y resultados. Journal of Spanish Language Teaching, 1(1), 101-115. https://doi.org/10.1080/23247797.2014.898516

Campillo-Ferrer, J.-M., Miralles-Martínez, P., y Sánchez-Ibáñez, R. (2020). CLIL teachers' views on cognitive development in primary education. Palgrave Communications, 6(1). https://doi.org/10.1057/s41599-020-0480-x

Camps, A. (2003). Secuencias didácticas para aprender a escribir. Graó.

Canales, A. B. (2010). Linguistic, pragmatic and functional specialty languages. Didactic and methodological implications. Revista Española de Lingüística Aplicada, 9, 72-85.

Caro Valverde, M. T., De Vicente-Yagüe Jara, M. I., y Valverde González, M. T. (2018). Teacher perception of methodological habits for informal argumentation in text commentary. Revista Española de Pedagogía, 76(270), 273-293. https://doi.org/10.22550/REP76-2-2018-04

Casado Velarde, M.(1993). Introducción a la gramática del texto del español. Arco Libros.

Casado Velarde, M. (2000). Lingüística y gramática del texto: Su articulación interdisciplinar. RILCE: Revista de filología hispánica. https://dialnet.unirioja.es/servlet/articulo?codigo=108088

Casassus, J., Froemel, J., Palafox, J.C., y Cusato, S. (1998). Primer Estudio Internacional Comparativo sobre Lenguaje, Matemática y Factores Asociados en Tercero y Cuarto Grado. Laboratorio Latinoamericano de Evaluación de la Calidad de la Educación. UNESCO.

Cassany, D., Luna, M., y Sanz, G. (2003). Enseñar lengua (9na.). Graó.

Centro Virtual Cervantes. (2009a). CVC. Diccionario de términos clave de ELE. Competencia discursiva. CVC. Centro Virtual Cervantes; Instituto Cervantes. https://cvc.cervantes.es/ensenanza/biblioteca_ele/diccio_ele/diccionario/competenciadiscursi va.htm 
Centro Virtual Cervantes. (2009b). CVC. Diccionario de términos clave de ELE. Enfoque comunicativo. CVC. Centro Virtual Cervantes; Instituto Cervantes. https://cvc.cervantes.es/ensenanza/biblioteca_ele/diccio_ele/diccionario/enfoquecomunicativ o.htm

Cisneros Estupiñán, M., Rojas García, I., y Olave Arias, G. (2016). Didáctica de la lengua materna en Colombia: Currículos y visiones docentes. Universidad Tecnológica de Pereira. http://ri.conicet.gov.ar/handle/11336/108539

Clares, P. M., y Morga, N. G. (2019). El dominio de competencias transversales en Educación Superior en diferentes contextos formativos. Educação e Pesquisa, 45. https://doi.org/10.1590/s16784634201945188436

Consejo de Europa. (2003). Common European Framework for Languages: Learning, Teaching, Assessment (2. ed). Anaya.

Cornieles, L. (2011). El punto de vista estudiantil en la construcción de un diagnóstico de necesidades y sus valoraciones en el área de la pedagogía del discurso. Letras, 53(85), 31-40.

Cortes Bueno, E. (2017). Strategies for the teaching of academic language. Proficiency from the instruction of oral skills. Foro De Profesores De E-Le, 13, 61-72. https://doi.org/10.7203/foroele.13.10838

Cox, C. B., y Montgomery, C. (2019). A study of 21st century skills and engagement in a university Spanish foreign language classroom. Foreign Language Annals, 52(4), 822-849. https://doi.org/10.1111/flan.12426

De Pablos-Ortega, C. (2016). Pragmática en la formación de profesores de Español como segunda lengua. Journal of Spanish Language Teaching, 3(2), 171-188. https://doi.org/10.1080/23247797.2016.1251783

De Zarobe, Y. R., y Zenotz, V. (2015). Reading strategies and CLIL: the effect of training in formal instruction. Language Learning Journal, 43(3), 319-333. https://doi.org/10.1080/09571736.2015.1053284

Del Valle Revuelta, S. (2018). Aspects of the communicative competence that can be worked with television programs. Tonos Digital, 35.

Dona, E., Stover, S., y Broughton, N. (2014). Modern languages and distance education: Thirteen Days in the Cloud. Turkish Online Journal of Distance Education, 15(3), 155-170. https://doi.org/10.17718/tojde.91273

Elola, I., y Oskoz, A. (2016). Supporting Second Language Writing Using Multimodal Feedback. Foreign Language Annals, 49(1), 58-74. https://doi.org/10.1111/flan.12183

Enkin, E., y Correa, M. (2018). Evaluating learner and teacher perceptions of program outcomes in the foreign language major. Electronic Journal of Foreign Language Teaching, 15(1), 66-80.

Enkin, E., y Mejias-Bikandi, E. (2017). The effectiveness of online teaching in an advanced Spanish language course. International Journal of Applied Linguistics, 27(1), 176-197. https://doi.org/10.1111/ijal.12112

Escagedo, C. P. (2019). The role of the listener in the acquisition of conversational competence in Spanish as a Foreign Language (SFL): Didactic proposal for teaching conversational listener's 
responses to Italian students of SFL C1 level. Revista Española de Lingüística Aplicada, 32(1), 220-247. https://doi.org/10.1075/resla.17048.pas

Escobar Urmeneta, C. (2013). Learning to become a CLIL teacher: Teaching, reflection and professional development. International Journal of Bilingual Education and Bilingualism, 16(3), 334-353. https://doi.org/10.1080/13670050.2013.777389

Espinosa Guerri, G. (2019). Drawing conversations in ELE: the teaching of the Spanish turn-taking system. Foro De Profesores De E-Le, 15, 105-125. https://doi.org/10.7203/foroele.15.14860

Estrada Chichón, J. L., y Zayas Martínez, F. (2019). Future teachers' beliefs on grammar in language teaching. Enunciacion, 24(2), 152-168. https://doi.org/10.14483/22486798.14838

Farías, M., Obilinovic, K., y Orrego, R. (2007). Implications of Multimodal Learning Models for foreign language teaching and learning. Colombian Applied Linguistics Journal, 9, 174-199.

Farías, M., y Véliz, L. (2019). Multimodal Texts in Chilean English Teaching Education: Experiences From Educators and Pre-Service Teachers. Profile Issues in Teachers` Professional Development, 21(2), 13-27. https://doi.org/10.15446/profile.v21n2.75172

Ferrerira Nobre, A. M. F., Barros, D. M. V., Mora, A. C., y Burgués, A. S. (2019). DK eLearning grid: Optimization of teaching practice in online environment. Revista de Educación a Distancia, 1(59). https://doi.org/10.6018/red/59/03

Flick, U. (2014). La gestión de la Calidad en Investigación Cualitativa. Morata.

Flotts, M. P., Manzi, J., Jiménez, D., Abarzúa, A., Cayuman, C., y García, M. J. (2015). Informe de resultados Tercer Estudio Regional Comparativo y Explicativo. Logros de aprendizaje. OREALCUNESCO.

Fricke, S. (2018). Semantic Scholar. Journal of the Medical Library Association, 106(1), 145-147. https://doi.org/10.5195/jmla.2018.280

Galván, L. (2006). Reading poetry in literary education: Notes on method. Revista de Literatura, 68(135), 41-75.

García, R., y Matos Moquete, M. (2013). Producto (interno)-Educando. yumpu.com. https://www.yumpu.com/es/document/read/14485585/producto-interno-educando

García Retana, J. Á. (2011). Modelo educativo basado en competencias: Importancia y necesidad. Revista Electrónica Actualidades Investigativas en Educación, 11(3), 1-24.

García-Dussán, É. (2016). Sobre el misterio estético en el texto literario: Claves para una pedagogía de la literatura. La Palabra, 28, 141-154.

Garrote, M., Alonso, I., y Galetti, E. (2018). Frequently Reported Practices in Communicative Language Teaching: An exploratory study at Secondary Schools and Official Schools of Languages in the Madrid region. Rael-Revista Electronica De Lingüística Aplicada, 17(1), 45-61.

González Cobas, J. (2019). Quixote and phraseology: A proposal for teaching Spanish. Didáctica. Lengua y Literatura, 31, 191-216. https://doi.org/10.5209/dida.65948

González Martínez, J., Espuny Vidal, C., y Gisbert Cervera, M. (2015). Learning language or not learning language. The acquisition of the communicative competence in secondary education in a highly technological environment. A study from Catalonia (Spain). Revista Complutense de Educación, 26(1), 141-160. https://doi.org/10.5209/rev_RCED.2015.v26.n1.42814 
Grossman, D. (2008). Democracia, educación para la ciudadanía e inclusión: Un enfoque multidimensional. Perspectivas, 38(1), 45-60.

Grupo Iberoamericano de PISA. (2009). Iberoamérica en PISA 2006. Informe regional. Santillana.

Gurzynski-Weiss, L. (2016a). Factors Influencing Spanish Instructors' In-Class Feedback Decisions. Modern Language Journal, 100(1), 255-275. https://doi.org/10.1111/modl.12314

Gurzynski-Weiss, L. (2016b). Spanish instructors' operationalisation of task complexity and task sequencing in foreign language lessons. Language Learning Journal, 44(4), 467-486. https://doi.org/10.1080/09571736.2015.1015151

Gutiérrez Arvizu, M. N. (2020). L2 Vocabulary Acquisition through Narratives in an EFL Public Elementary School. IAFOR Journal of Education, 8(1), 115-128. https://doi.org/10.22492/ije.8.1.07

Gutiérrez Ospina, A. T., y Fernan, F. (2020). Analysis of narrative production in children from the proposals of Karmiloff, Karmiloff and Owens. Sophia-Educación, 16(1), 110-119. https://doi.org/10.18634/sophiaj.16v.1i.1008

Gutiérrez Tapias, M. (2018). Estilos de aprendizaje, estrategias para enseñar. Su relación con el desarrollo emocional y "aprender a aprender». Tendencias pedagógicas, 31, 83-96.

Haché, A., y Montenegro, L. (2013). Tarea 2.1: Identificación de avances en las disciplinas científicas y literarias asociadas al área de Lengua Española que deben ser tomados en cuenta para la revisión y actualización curricular.

Harper, F., y Hamer, M. (2006). Facilitating access to further languages: Trialling materials combining parallel texts with reflective learning. Language Learning Journal, 34(1), 14-24. https://doi.org/10.1080/09571730685200191

Harper, F., Green, H., y Fernández-Toro, M. (2018). Using screencasts in the teaching of modern languages: Investigating the use of Jing ${ }^{\circledR}$ in feedback on written assignments. Language Learning Journal, 46(3), 277-292. https://doi.org/10.1080/09571736.2015.1061586

Harzing, A.-W., y Alakangas, S. (2016). Google Scholar, Scopus and the Web of Science: A longitudinal and cross-disciplinary comparison. Scientometrics, 106(2), 787-804. https://doi.org/10.1007/s11192-015-1798-9

Hernández, A. A. (2018). Edublogs in foreign language teaching: Integrating language and culture. Revista Letral, 20, 86-101. https://doi.org/10.30827/RL.v1i20.7829

Hernández Liechti, A. (2017). Teach E / LE to the first generation of digital natives. Foro De Profesores De E-Le, 13, 165-176. https://doi.org/10.7203/foroele.13.10793

Hernández López, N. (2018). The comic in the SFL: an experience for teaching Geography of Spain. Foro De Profesores De E-Le, 14, 121-130. https://doi.org/10.7203/foroele.14.13341

Hernández, T. A. (2010). Promoting Speaking Proficiency through Motivation and Interaction: The Study Abroad and Classroom Learning Contexts. Foreign Language Annals, 43(4), 650-670. https://doi.org/10.1111/j.1944-9720.2010.01107.x

Hertel, T. J., y Harrington, S. (2015). Promoting cultural and linguistic competence with documentary film in Spanish. Hispania, 98(3), 549-569. https://doi.org/10.1353/hpn.2015.0094 
Hornillos, R. C., y Roa, J. D. V. (2015). The music, a tool in teaching Spanish as a foreign language through the application of new technologies. Porta Linguarum, 23, 139-151.

Hurd, S., Beaven, T., y Ortega, A. (2001). Developing Autonomy in a Distance Language Learning Context: Issues and Dilemmas for Course Writers. System, 29(3), 341-355. https://doi.org/10.1016/S0346-251X(01)00024-0

lizuka, T. (2019). Task-based needs analysis: Identifying communicative needs for study abroad students in Japan. System, 80, 134-142. https://doi.org/10.1016/j.system.2018.11.005

Internet Live Stats-Internet Usage y Social Media Statistics. (s. f.). Recuperado 10 de septiembre de 2020, de https://www.internetlivestats.com/

Khong, H. K., Cheah, S. E., Wan, N. N., y Vellon, S. M. (2018). Does the adapted version of The Amazing Race (AVOTAR) benefit Spanish language learning among technical students? An empirical study. Revista Española De Lingüística Aplicada, 31(1), 159-196. https://doi.org/10.1075/resla.15019.kho

Kissau, S., y Algozzine, B. (2017). Effective Foreign Language Teaching: Broadening the Concept of Content Knowledge. Foreign Language Annals, 50(1), 114-134. https://doi.org/10.1111/flan.12250

Krepel, R., y Sinclair, E. (2019). Motivating Secondary Students to Speak in Spanish Outside of Structured Classroom Activities. Journal of Language Teaching and Learning, 9(1), 115-127.

Lacorte, M. (2007). Interaction and contexts in the learning and teaching of Spanish as a second language. Spanish in Context, 4(1), 73-98. https://doi.org/10.1075/sic.4.1.04lac

Lanseros Sánchez, R., y Sánchez García, M. R. (2017). Poetry for advanced level of Spanish as a foreign language in the Official Schools of Languages: A didactic proposal. Espiral-Cuadernos Del Profesorado, 10(21), 90-99.

Lanseros Sánchez, R., y Sánchez García, M. R. (2018). Didactic Exploitation of Poetry in the Classroom of Spanish as a Foreign Language. Proposal of Work for the Advanced Level of the Official Schools of Languages from a Poem by Clara Janes. Didáctica Lengua y Literatura, 30, 105-115. https://doi.org/10.5209/DIDA.61957

Lantolf, J. P. (2011). Dynamic assessment in the classroom: Vygotskian praxis for second language development. Language Teaching Research, 15(1), 11-33. https://doi.org/10.1177/1362168810383328

Lee, L. (2014). Digital news stories: Building language learners' content knowledge and speaking skills. Foreign Language Annals, 47(2), 338-356. https://doi.org/10.1111/flan.12084

Lenkaitis, C. A., Calo, S., y Venegas Escobar, S. (2019). Exploring the intersection of language and culture via telecollaboration: Utilizing videoconferencing for intercultural competence development. International Multilingual Research Journal, 13(2), 102-115. https://doi.org/10.1080/19313152.2019.1570772

Level Hernández, M. B. (2009). Textos escolares: Oralidad, lectura y escritura. Educere, 13(44), 131138.

Llovet Vila, X. (2006). El enfoque integrado de contenidos y lenguas extranjeras (EICLE): Un reto posible. Congreso Nacional: 2006, año del español en Noruega: un reto posible, 11. 
Lobachev, S. (2008). Top languages in global information production. Partnership: The Canadian Journal of Library and Information Practice and Research, 3(2), Article 2. https://doi.org/10.21083/partnership.v3i2.826

Loewen, S., Isbell, D. R., y Sporn, Z. (2020). The effectiveness of app-based language instruction for developing receptive linguistic knowledge and oral communicative ability. Foreign Language Annals, 53(2), 209-233. https://doi.org/10.1111/flan.12454

Lomas García, C. (1999). Cómo enseñar a hacer cosas con las palabras: Teoría y práctica de la educación lingüística. Paidós.

López Quero, S. (2017). The Teaching of the Grammatical Component in ELE Based on the Values of "se": Methodological suggestions for the professor. Moenia-Revista Lucense de Lingüística y Literatura, 23, 697-716.

López-García-Torres, R., y Saneleuterio, E. (2020). Axiological study of the standard curriculum of spanish language and literature. Revista Complutense de Educación, 31(3), 307-317. https://doi.org/10.5209/rced.63205

López-Jiménez, M. D. (2018). Diversity and sociocultural contents in textbooks for teaching Spanish as an L2. Revista de Educación, 2018(382), 11-31. https://doi.org/10.4438/1988-592X-RE-2018$382-390$

Lyster, R., y Ballinger, S. (2011). Content-based language teaching: Convergent concerns across divergent contexts. Language Teaching Research, 15(3), 279-288. https://doi.org/10.1177/1362168811401150

Marchi, B. U., y Las, C. G. (2013). Teaching/learning languages in primary education degree. Porta Linguarum, 20, 223-237.

Martín, L., y Cabrera, A. (2018). Available lexicon in three interest centers among sfl learners. Ikala, 23(3), 505-517. https://doi.org/10.17533/udea.ikala.v23n03a07

Martínez Canton, C. I., y Ruiz Fabo, P. (2019). Literary Tool Evaluation as a Way of Introducing Digital Humanities in University Courses. The "Distant Poetry" case. Didáctica. Lengua y Literatura, 31, 171-190. https://doi.org/10.5209/dida.65947

Martínez, M. C. M. (2016). Los géneros desde una perspectiva socio-enunciativa. La noción de contexto integrado. Revista Latinoamericana de Estudios del Discurso, 13(2), 21-40. https://doi.org/10.35956/v.13.n2.2013.p.21-40

Martínez-Lirola, M., y Martínez-Lirola, M. (2019). Una propuesta didáctica para introducir la educación para la ciudadanía global en la enseñanza universitaria. Revista Electrónica Educare, 23(2), 286305. https://doi.org/10.15359/ree.23-2.15

McMullin, K. (2016). Cooperation and Autonomy in Language Learning: An Application of the Collage Method. Porta Linguarum: revista internacional de didáctica de las lenguas extranjeras, 25, 93103.

Ministerio de Educación, Cultura y Deporte. (2014). PISA 2012. Programa para la Evaluación Internacional de los Alumnos. Informe español. Resultados y contexto. Secretaría General Técnica. Subdirección General de Documentación y Publicaciones. 
Ministerio de Educación, Cultura y Deporte. (2016). PISA 2015. Programa para la Evaluación Internacional de los Alumnos. Informe español. Secretaría General Técnica. Subdirección General de Documentación y Publicaciones.

Ministerio de Educación de España. (2010). PISA 2009. Programa para la Evaluación Internacional de Alumnos de la OCDE. Informe español. Ministerio de Educación. Instituto de Evaluación. MINERD

Ministerio de Educación de la República Dominicana. (2016). Bases de la Revisión y Actualización Curricular. MINERD

Ministerio de Educación de la República Dominicana. (2019). Naturaleza de las Áreas Curriculares. Versión Preliminar para Revisión y Aportes. MINERD

Monarca, C. R., y Llancao, C. Q. (2016). Illocutionary potential of verb conjugation in Spanish. A critical and propositional paraphrase of "La enseñanza del verbo en la educación básica" by Hernán Urrutia. Estudios Pedagogicos, 42(ESPECIAL), 137-149.

Morán López, P. M. (2016). E-learning in the ELE classroom: From the blogs to the beacon technology. Opcion, 32(Special Issue 11), 973-989.

Moranski, K., y Henery, A. (2017). Helping Learners to Orient to the Inverted or Flipped Language Classroom: Mediation via Informational Video. Foreign Language Annals, 50(2), 285-305. https://doi.org/10.1111/flan.12262

Navarro, F., Reyes, N. Á., Tapia-Ladino, M., Cristovão, V. L. L., Moritz, M. E. W., Cardona, E. N., y Bazerman, C. (2016). A historical and contrastive account of higher education reading and writing studies published in Latin America. Revista Signos, 49, 100-126. https://doi.org/10.4067/S0718-09342016000400006

Nikleva, D. G. (2018). The visual and musical codes in the framework of semiological competition in Spanish as a foreign language. Revista Española De Lingüística Aplicada, 31(2), 495-519. https://doi.org/10.1075/resla.15026.nik

Noreña Peña, D. M. (2019). Estrategias de Autorregulación en el Aprendizaje del Inglés como Lengua Extranjera [Universidad de Antioquia]. http://bibliotecadigital.udea.edu.co/bitstream/10495/15067/1/NorenaDiana_2020_Estrategi asAutorregulacionIngles.pdf

OECD. (2019). PISA 2018 Results (Volume I): What Students Know and Can Do. OECD. https://doi.org/10.1787/5f07c754-en

Organización de las Naciones Unidas para la Educación, la Ciencia y la Cultura, y Oficina Regional de Educación para América Latina y el Caribe. (2020). ¿Qué se esperará después de los estudiantes de América Latina y el Caribe? Análisis curricular del Estudio Regional Comparativo y Explicativo (ERCE 2019). OREALC-UNESCO.

Pellicer-Sánchez, A. (2015). Developing automaticity and speed of lexical access: The effects of incidental and explicit teaching approaches. Journal of Spanish Language Teaching , 2 (2) pp. 126-139. (2015), 2(2), 126-139. https://doi.org/10.1080/23247797.2015.1104029

Pérez Niño, D. F. (2010). The Role of Music in Young Learners' Oral Production in English. Profile Issues in Teachers` Professional Development, 12(1), 141-157. 
Pérez-Valverde, C., y Ruiz R. (2012). Paving the way towards the ECTS system: Self-assessment, metacognition, and professional competence in a literature course for FL teachers. Porta Linguarum, 17, 67-77.

Pérez-Valverde, C. y Ruiz, R. (2014). Narratives of professional identity in the training of foreign language teachers. Andamios, 11(24), 215-234. https://doi.org/10.29092/uacm.v11i24.240

López-García, M.P. (2017). The teaching of Spanish to heritage speakers in partial and total immersion: Effective factors of learning in heterogeneous classrooms. Tejuelo-Didáctica de la Lengua y la Literatura, 26, 113-142. https://doi.org/10.17398/1988-8430.26.113

Piriyasurawong, P. (2019). Active learning using ARCS motivation on social cloud model to enhance communication skills in foreign language. TEM Journal, 8(1), 290-297. https://doi.org/10.18421/TEM81-40

Pizarro, M. A. (2013). Primary education degrees in Spain: Do they fulfil the linguistic and pedagogic needs of future teachers? Vigo International Journal of Applied Linguistics, 10(1), 9-27.

Plo, R., Hornero, A., y Mur-Dueñas, P. (2014). Implementing the teaching/learning of oral skills in secondary education in Aragón: Gauging teachers' attitudes, beliefs and expectations. International Journal of English Studies, 14(1), 55-77. https://doi.org/10.6018/ijes/14/1/175041

Pomata García, J. A., y Díaz Ayuga, J. M. (2017). ICT and gamificacion in the teaching of Spanish as a foreign language: Situation and lines of action for Japanese universities. Cuadernos Canela, 28, 79-101.

Prado Aragonés, J.2004). Didáctica de la lengua y la literatura para educar en el siglo XXI. La muralla.

Price, D. J. de S. (1986). Little Science, Big Science and Beyond.

Rachels, J., y Rockinson-Szapkiw, A. (2018). The effects of a mobile gamification app on elementary students' Spanish achievement and self-efficacy. Assisted Language Learning, Query date: 202007-11 08:50:02. https://www.tandfonline.com/doi/abs/10.1080/09588221.2017.1382536

Rädiker, S., y Kuckartz, U. (2020). Trabajar con información bibliográfica y crear revisiones de la literatura. En Análisis de datos cualitativos con MAXQDA: Texto, audio, video (pp. 203-216). MAXQDA Press. https://doi.org/10.36192/978-3-948768003_14

Ramírez Guerrero, V., Fonseca Sanz, A., y Rodríguez Aguilar, Y. (2019). Understanding written texts from promotion and animation to reading in primary school. Dilemas Contemporáneos-Educación Política y Valores, 7(1), 34.

Ramos-González, N. M., y Rico-Martín, A. M. (2014). Analysis of expression of politeness of international RTVE for teaching Spanish as a foreign language. RLA, 52(1), 79-103. https://doi.org/10.4067/s0718-48832014000100005

Remesal, A., y Brown, G. T. L. (2015). Conceptions of assessment when the teaching context and learner population matter: Compulsory school versus non-compulsory adult education contexts. European Journal of Psychology of Education, 30(3), 331-347. https://doi.org/10.1007/S10212014-0236-3

Rodrigo, I. H. (2017). Assisting language learning with new technologies: A case of spanish degrees facing a European educational change process. CALL-EJ, 18(1), 40-61. 
Rodrigues, T. L. (2019). The Variation in the Representation of the Verbal Complement and the Teaching of BP to Spanish Speakers: An Analysis of Didactic Materials of PFL. Revista De Estudos Da Linguagem, 27(1), 49-72. https://doi.org/10.17851/2237-2083.27.1.49-72

Rodríguez Arocho, W. C. (2018). La alfabetización desde una perspectiva crítica: Los aportes de Vygotski, Freire y Martín Baró. Actualidades Investigativas en Educación, 19(1), 25. https://doi.org/10.15517/aie.v19i1.35569

Rodríguez, G. O. G., y Hernández, C. A. G. (2014). Competencias ciudadanas: Consideraciones desde el concepto de ciudadanía. Plumilla Educativa, 13(1), 373-396. https://doi.org/10.30554/plumillaedu.13.416.2014

Rodríguez, M. N. R., y García-Perera, D. (2016). El microrrelato como material didáctico en la adquisición de la gramática en ele. Revista de Lingüística y Lenguas Aplicadas, 11, 61-70. https://doi.org/10.4995/rlyla.2016.3936

Rodríguez Mata, L. (2019). The Cultural Dimension of the Poem «Night", written by Josefina de la Torre: Didactic Proposal for the SSL classroom. Guiniguada, 28, 111-121.

Rodríguez-Gonzalo, C., y Zayas, F. (2017). THE RELATIONSHIP BETWEEN GRAMMATICAL KNOWLEDGE AND DISCOURSE PRACTICE IN LANGUAGE LEARNING: AN ANALYSIS OF THE ADJECTIVE. Caplletra, 63, 245-277. https://doi.org/10.7203/Caplletra.63.10401

Rojas Moreno, W. E. (2019). Popular Culture to Teach Spanish as a Foreign Language. Cuadernos De Lingüística Hispanica, 34, 177-+. https://doi.org/10.19053/0121053X.n34.2019.9373

Ruiz-Funes, M. (2001). Task representation in foreign language reading-to-write. Foreign Language Annals, 34(3), 226-234.

Russell, V., y Curtis, W. (2013). Comparing a large- and small-scale online language course: An examination of teacher and learner perceptions. Internet and Higher Education, 16(1), 1-13. https://doi.org/10.1016/j.iheduc.2012.07.002

Sánchez M., González, A. J. y García Pérez, R. (2002). Competencia retórica. Una propuesta para interpretar las dificultades de comprensión. Psicothema, 14(1), 77-85.

Sánchez-Vizcaíno, M. C., y Mora, M. C. F. (2018). Music videos and emotions applied in learning Spanish as a Foreign Language. Círculo de Lingüística Aplicada a la Comunicación, 78, 255-286. https://doi.org/10.5209/CLAC.64381

Sanjuan Álvarez, M., y del Moral Barriguete, C. (2019). Educational principles for the teaching of vocabulary in Spanish as the language of instruction for immigrant students. Didáctica. Lengua $Y$ Literatura, 31, 99-116. https://doi.org/10.5209/dida.65940

Santos Díaz, I. C. (2017). Word organization in the mind of native speakers and foreign language learners (English and French). Pragmalingüística, 25, 603-617.

Santos, J. M. R. (2019). Cultural rhetoric: Contributions for the training of teachers of Spanish as a foreign language. Tonos Digital, 2019(36).

Secchi, D. (2017). The teaching of attenuation in E / LE based on the analysis of a real corpus. Foro De Profesores De E-Le, 13, 247-257. https://doi.org/10.7203/foroele.13.10835

Sedano Cuevas, B. (2017). Attending to the specific needs and demands of students in a globalised world: The case of a "Spanish for travelling" MOOC. Ried-Revista Iberoamericana de Educación a Distancia, 20(1), 161-182. https://doi.org/10.5944/ried.20.1.16692 
Serafini, E. J., y Torres, J. (2015). The Utility of Needs Analysis for Nondomain Expert Instructors in Designing Task-Based Spanish for the Professions Curricula. Foreign Language Annals, 48(3), 447-472. https://doi.org/10.1111/flan.12150

Sessarego, C. (2016). A Discourse-Pragmatic Approach to Teaching Indicative/Subjunctive Mood Selection in the Intermediate Spanish Language Class: New Information versus Reformulation. Hispania, 99(3), 392-406. https://doi.org/10.1353/HPN.2016.0067

Showstack, R. E. (2016). La pragmática transcultural de los hablantes de herencia de español: Análisis e implicaciones pedagógicas. Journal of Spanish Language Teaching, 3(2), 143-156.

Simich-Dudgeon, C. (2009). Antología de textos de didáctica del español. Enseñanza de una segunda lengua a inmigrantes. CVC. Centro Virtual Cervantes; Instituto Cervantes. https://cvc.cervantes.es/ensenanza/biblioteca_ele/antologia_didactica/inmigracion/simich05. $\mathrm{htm}$

Simon, F. R. (2018). A cognitive analysis of the obligation modal periphrasis: The equivalence between "deber plus infinitivo" and "tener que plus infinitivo". Círculo De Lingüística Aplicada a La Comunicación, 73, 217-242. https://doi.org/10.5209/CLAC.59067

Sparks, R. L., Patton, J., y Luebbers, J. (2019). Individual differences in L2 achievement mirror individual differences in L1 skills and L2 aptitude: Crosslinguistic transfer of L1 to L2 skills. Foreign Language Annals, 52(2), 255-283. https://doi.org/10.1111/flan.12390

Strauss, A. L., Corbin, J., y Zimmerman, E. (2002). Bases de la investigación cualitativa: Técnicas y procedimientos para desarrollar la teoría fundamentada. Universidad de Antioquía.

Swanson, P. (2017). The future of K-12 teacher education: Spanish and Portuguese. Hispania, 100(5), 261-267. https://doi.org/10.1353/hpn.2018.0064

Taguchi, N., Gomez-Laich, M. P., y Arrufat-Marques, M.-J. (2016). Comprehension of Indirect Meaning in Spanish as a Foreign Language. Foreign Language Annals, 49(4), 677-698. https://doi.org/10.1111/flan.12230

Taourite, F., y Ruiz-Cecilia, R.(2020). Perceptions and application of task-based language teaching among primary school EFL teachers in Spain. Universal Journal of Educational Research, 8(4), 1289-1298. https://doi.org/10.13189/ujer.2020.080420

Tedick, D. J., y Young, A. I. (2018). Two-way immersion students' home languages, proficiency levels, and responses to form-focused instruction. International Journal of Bilingual Education and Bilingualism, 21(3), 303-318. https://doi.org/10.1080/13670050.2017.1383354

Terradez Gurrea, M. (2017). The incorporation of the variable rule in the teaching of E / LE. Foro De Profesores De E-Le, 13, 283-290. https://doi.org/10.7203/foroele.13.10853

Tobón, S. (2006). Competencias, calidad y educación superior. Coop. Editorial Magisterio.

Tobón, S. (2007). El enfoque complejo de las competencias y el diseño curricular por ciclos propedéuticos. Acción Pedagógica, 16, 14-28.

Tolchinsky, L., Johansson, V., y Zamora, A. (2002). Text openings and closings in writing and speech: Autonomy and differentiation. Written Language and Literacy, 5(2), 219-253. https://doi.org/10.1075/WLL.5.2.05TOL 
Torres-Guzmán, M. E. (2011). Methodologies and teacher stances: How do they interact in classrooms? International Journal of Bilingual Education and Bilingualism, 14(2), 225-241. https://doi.org/10.1080/13670050.2010.539675

Trujillo Sáez, F., Salvadores Merino, C., y Gabarrón Pérez, Á. (2019). Tecnología para la enseñanza y el aprendizaje de lenguas extranjeras: Revisión de la literatura. Revista Iberoamericana de Educación a Distancia, 22(1), 153-169. http://dx.doi.org/10.5944/ried.22.1.22257

Urrútia, G., y Bonfill, X. (2010). Declaración PRISMA: Una propuesta para mejorar la publicación de revisiones sistemáticas y metaanálisis. Medicina Clínica, 135(11), 507-511. https://doi.org/10.1016/j.medcli.2010.01.015

Valdés, H., Treviño, E., Acevedo, C. G., Castro, M., Carrillo, S., Costilla, R., Bogoya, D. y Pardo, C. (2008). Los aprendizajes de los estudiantes de América Latina y el Caribe. Resumen Ejecutivo del Primer Reporte de Resultados del Segundo Estudio Regional Comparativo y Explicativo. Laboratorio Latinoamericano de Evaluación de la Calidad de la Educación. UNESCO.

Valério, K. M., y Mattos, A. M. de A. (2018). Critical Literacy and the Communicative Approach: Gaps and Intersections. Revista Brasileira de Linguística Aplicada, 18(2), 313-338. https://doi.org/10.1590/1984-6398201812252

Van Compernolle, R. A., Gomez-Laich, M. P., y Weber, A. (2016). Teaching L2 Spanish Sociopragmatics Through Concepts: A Classroom-Based Study. Modern Language Journal, 100(1), 341-361. https://doi.org/10.1111/modl.12318

Verdessi Hoy, G. M. (2015). Reflection and proposal of a learning object for the teaching of Spanish. Opcion, 31(Special Issue 2), 1129-1150.

Villalba Ibánez, C. (2019). Marketing and publicity in the Spanish as Foreign Language classroom. A didactic proposal. Foro De Profesores De E-Le, 15, 267-275. https://doi.org/10.7203/foroele.15.14858

Yáñez-Prieto, M.-C. (2014). Sense and subjectivity: Teaching literature from a sociocultural perspective. Language and Sociocultural Theory, 1(2), 176-203. https://doi.org/10.1558/lst.v1i2.179

Yepes, G., y Krishnamurthy, R. (2010). Corpus linguistics and second language acquisition-the use of ACORN in the teaching of Spanish Grammar. Lebende Sprachen, Query date: 2020-07-11 08:50:02. https://www.degruyter.com/view/journals/les/55/1/article-p108.xml

Zevallos Polo, D. S., Arcos Araujo, N. P., y Ripoll Salceda, J. C. (2017). The simple view of reading in 4th grade grade students from a public school in Quito. Alteridad-Revista De Educación, 12(1), 115122. https://doi.org/10.17163/alt.v12n1.2017.10

Ziegler, N. A., y Moeller, A. J. (2012). Increasing Self-Regulated Learning Through the LinguaFolio. Foreign Language Annals, 45(3), 330-348. https://doi.org/10.1111/j.1944-9720.2012.01205.x 